\title{
FRAMED AND MW-TRANSFERS FOR HOMOTOPY MODULES
}

\author{
ALEXEY ANANYEVSKIY AND ALEXANDER NESHITOV
}

\begin{abstract}
In the paper we use the theory of framed correspondences to construct MilnorWitt transfers on homotopy modules. As a consequence we identify the zeroth stable $\mathbb{A}^{1}$ homotopy sheaf of a smooth variety with the zeroth homology of the corresponding MWmotivic complex and prove that the hearts of the homotopy $t$-structures on the stable $\mathbb{A}^{1}$ derived category and the category of Milnor-Witt motives are equivalent. We also show that a homotopy invariant stable linear framed Nisnevich sheaf admits a unique structure of a presheaf with MW-transfers compatible with the framed structure.
\end{abstract}

\section{INTRODUCTION}

The theory of framed correspondences was introduced by Voevodsky in [16] and developed by Garkusha and Panin [9, 10]. This theory can be seen as an enhancement of Voevodsky's theory of presheaves with transfers [12] adjusted to the setting of motivic homotopy theory. Presheaves with transfers have wrong-way maps (transfers) along finite surjective morphisms $Z \rightarrow X$; for framed presheaves one has transfers along finite surjective morphisms $Z \rightarrow X$ equipped with the additional data: (1) a factorization $Z \stackrel{i}{\rightarrow} \mathbb{A}^{n} \times X \stackrel{p}{\rightarrow} X$, where $i$ is a regular embedding and $p$ is the projection, and (2) trivialization of the normal bundle $N_{i}$. Every presheaf on the category of smooth varieties over a field that can be factored through the motivic stable homotopy category has a canonical structure of a presheaf with framed transfers.

Calmès and Fasel introduced another enhancement of presheaves with transfers, the so-called presheaves with Milnor-Witt transfers [4]. These presheaves have wrong-way maps along the finite surjective maps equipped with a certain unramified quadratic datum (that endows the morphism with a kind of an orientation). The theory was subsequently developed in $[5,7,8]$.

In this paper we study the relations between the theories based on framed and Milnor-Witt correspondences over an infinite perfect field $k$ of characteristic different from 2 . We construct a "forgetful" functor $\mathbb{Z} \mathrm{F}_{*}(k) \rightarrow \widehat{\operatorname{Cor}}(k)$ (see Proposition 7.8; there is another construction given in [7]) and obtain the following comparison results.

1.1. Theorem (Theorem 8.8). For a smooth variety $X$ the forgetful functor induces an isomorphism $\mathrm{H}_{0}^{\mathbb{Z F}}(X) \cong \mathrm{H}_{0}^{\mathrm{MW}}(X)$ of the zeroth $\mathbb{Z} \mathrm{F}$ and $\mathrm{MW}$-homology sheaves.

Recall that $\pi_{0}\left(\Sigma_{\mathbb{P 1}}^{\infty} X_{+}\right)_{0} \cong \mathrm{H}_{0}^{\mathbb{Z F}}(X)$ by [9, Theorem 11.1 and Corollary 11.3], so this theorem provides a reasonable description for the zeroth stable motivic homotopy sheaf of $X$. A similar answer with $X$ being a smooth projective variety was obtained by different techniques in [3, Theorem 4.3.1] and [1, Theorem 1.2], see also [1, Theorem 1.3] for the case of a smooth curve.

1.2. Corollary (Corollary 8.11). Let $M$ be a homotopy invariant stable $\mathbb{Z F}_{*}$-sheaf. Then $M$ admits a unique structure of a presheaf with $M W$-transfers compatible with the framed structure.

2010 Mathematics Subject Classification. 14F42.

Key words and phrases. Homotopy modules, framed correspondences, Milnor-Witt correspondences.

The first author is supported by Young Russian Mathematics award, by "Native towns", a social investment program of PJSC "Gazprom Neft", and by RFBR grant 18-31-20044. 


\subsection{Theorem (Theorem 8.12). The forgetful functors induce equivalences}

$$
\Pi_{*}^{\mathrm{MW}}(k) \stackrel{\simeq}{\rightarrow} \Pi_{*}^{\mathrm{fr}}(k) \stackrel{\simeq}{\longrightarrow} \Pi_{*}(k)
$$

between the categories of homotopy modules, framed homotopy modules and homotopy modules equipped with $M W$-transfers.

The category of homotopy modules [14, Section 5.2] can be identified with the heart of the homotopy $t$-structure on the stable $\mathbb{A}^{1}$-derived category (see, for example, [3, Theorem 3.3.3]). In a similar way one identifies the category $\Pi_{*}^{\mathrm{MW}}(k)$ with the heart of the homotopy $t$-structure on the category of MW-motives $\widetilde{\mathrm{DM}}(k)$. Thus we have the following corollary.

1.4. Corollary (Corollary 8.14). The forgetful functor $\widetilde{\mathrm{DM}}(k) \rightarrow \mathrm{D}_{\mathbb{A}^{1}}(k)$ induces an equivalence of the hearts of the homotopy t-structures.

The paper is organized as follows. In section 2 we recall the main definitions of the theory of framed correspondences and list important properties of presheaves with framed transfers that follow from [10]. In section 3 we construct framed transfers on a homotopy module and prove that every homotopy module has a unique structure of a stable $\mathbb{Z} \mathrm{F}_{*}$-sheaf such that the suspension isomorphisms agree with framed transfers. In section 4 we provide a description of framed transfers via a trivialization of the conormal sheaf for the support (see Corollary 4.9). In sections 5 and 6 we use the framed structure on homotopy modules to construct the pushforward map on cohomology groups of homotopy modules. For a map $f: X \rightarrow Y$ of smooth varieties with a closed subset $Z \subset X$ finite over $Y$ we locally construct a framed enhancement for $f$ giving a wrong-way framed correspondence then use Cousin complex to glue the induced maps into a pushforward map from the twisted cohomology of $X$ with support on $Z$ to the cohomology of $Y$ (Definition 6.3). The resulting pushforward maps coincide with the transfer maps constructed in $[13, \S 3,4]$, but we need the framed interpretation to prove a special case of the base change theorem 6.11. In section 7 we recall the main definitions of the theory of Milnor-Witt correspondences introduced in [4] and discuss the natural action of the framed correspondences on the Milnor-Witt ones. Then we use the pushforward maps introduced in section 6 to define an action of Milnor-Witt correspondences on the cohomology groups of homotopy modules. In Proposition 7.8 we construct a functor from the category of linear framed correspondences to the category of MW-correspondences. Such functor was also constructed in [7], but our approach is a different one and is based on the natural action of framed correspondences on the cohomology of Milnor-Witt K-theory. In the section 8 we prove that every homotopy module has a unique structure of a presheaf with Milnor-Witt transfers that agrees with the framed structure. Then we derive the two main results of the article: establish an isomorphism between the zeroth stable motivic homotopy sheaf of $X$ with the zeroth homology of the MW-motive of $X$ and show that the categories of homotopy modules, homotopy modules with MW-transfers and framed homotopy modules are equivalent.

Throughout the paper we employ the following notation. 


\begin{tabular}{l|l}
$k$ & an infinite perfect field of char $\neq 2$ \\
$\mathrm{Sm}_{k}$ & the category of smooth quasi-projective schemes over $k$ \\
$\mathrm{Shv} \bullet$ & the category of pointed Nisnevich sheaves of sets on $\mathrm{Sm}_{k}$ \\
$\mathrm{ShvAb}$ & the category of Nisnevich sheaves of abelian groups on $\mathrm{Sm}_{k}$ \\
$X_{+}$ & the pointed scheme $X \sqcup \mathrm{Spec} k$ considered as an object of Shv \\
$\mathbb{P}^{1}$ & the pointed sheaf represented by the pointed scheme $\left(\mathbb{P}^{1}, \infty\right)$ \\
$T$ & the quotient $\mathbb{A}^{1} / \mathbb{G}_{m}$ considered as an object of Shv \\
$\sigma: \mathbb{P}^{1} \rightarrow T$ & the composition $\mathbb{P}^{1} \rightarrow \mathbb{P}^{1} /\left(\mathbb{P}^{1}-0\right) \cong \mathbb{A}^{1} / \mathbb{G}_{m}=T$ \\
$\mathbb{P}^{\wedge n}, T^{n}$ & the $n$-fold smash power of the respective objects of Shv \\
$\sigma^{n}: \mathbb{P}^{\wedge n} \rightarrow T^{n}$ & the $n$-fold smash power of $\sigma$ \\
sheaf & Nisnevich sheaf
\end{tabular}

1.1. Acknowledgements. This work was initiated during the program "Algebro-Geometric and Homotopical Methods" at the Institut Mittag-Leffler in 2017. Authors would like to thank the organizers Eric Friedlander, Lars Hesselholt, and Paul Arne Østvaer.

\section{Homotopy invariant StABle $\mathbb{Z} F_{*}$-SHEAVES}

The notion of framed correspondences was introduced by Voevodsky in [16] and studied by Garkusha and Panin in [9]. We briefly recall the main definitions and constructions and refer the reader to [9] and [10] for the details.

2.1. Definition. For $X, Y \in \operatorname{Sm}_{k}$ and closed subsets $A \subset X$ and $B \subset Y$ let $\operatorname{Fr}(X / A, Y /(Y-B))$ be the set of equivalence classes of triples $(Z, U, g)$ where

(1) $Z \subset X$ is a closed subset such that $Z \cap A=\emptyset$,

(2) $U$ is an étale neighborhood of $Z$ in $X$,

(3) $g: U \rightarrow Y$ is a regular morphism such that $Z=g^{-1}(B)$.

The triples $(Z, U, g)$ and $\left(Z^{\prime}, U^{\prime}, g^{\prime}\right)$ are said to be equivalent if $Z=Z^{\prime}$ and there is an étale neighborhood $V$ of $Z$ in $U \times{ }_{X} U^{\prime}$ such that $g$ and $g^{\prime}$ coincide on $V$.

2.2. Lemma (Voevodsky; see also [9, Corollary 3.3]). For $X, Y \in \mathrm{Sm}_{k}$ and closed subsets $A \subset X$ and $B \subset Y$ there is a canonical bijection

$$
\operatorname{Hom}_{\text {Shv }}(X / A, Y /(Y-B)) \cong \operatorname{Fr}(X / A, Y /(Y-B)) .
$$

2.3. Definition. For $F, G \in \mathrm{Shv} \bullet$ put

$$
\operatorname{Fr}_{n}(F, G)=\operatorname{Hom}_{\text {Shv }}\left(F \wedge \mathbb{P}^{\wedge n}, G \wedge T^{n}\right)
$$

For $F, G, H \in \operatorname{Shv}_{\bullet}$ and $a \in \operatorname{Fr}_{n}(F, G), b \in \operatorname{Fr}_{m}(G, H)$ denote $b \circ a \in \operatorname{Fr}_{n+m}(F, H)$ the composition

$$
F \wedge \mathbb{P}^{\wedge n} \wedge \mathbb{P}^{\wedge m} \stackrel{a \wedge \text { id }}{\rightarrow} G \wedge T^{n} \wedge \mathbb{P}^{\wedge m} \stackrel{t w_{1}}{\rightarrow} G \wedge \mathbb{P}^{\wedge m} \wedge T^{n} \stackrel{b \wedge \text { id }}{\rightarrow} H \wedge T^{m} \wedge T^{n} \stackrel{t w_{2}}{\rightarrow} H \wedge T^{n} \wedge T^{m}
$$

where $t w_{1}$ permutes $\mathbb{P}^{\wedge m}$ with $T^{n}$ and $t w_{2}$ permutes $T^{m}$ with $T^{n}$.

2.4. Definition. For $X, Y \in \operatorname{Sm}_{k}$ we write $\operatorname{Fr}_{n}(X, Y)$ for $\operatorname{Fr}_{n}\left(X_{+}, Y_{+}\right)$. The set $\operatorname{Fr}_{n}(X, Y)$ is pointed by the trivial morphism sending everything to the distinguished point. Lemma 2.2 in this particular case identifies $\operatorname{Fr}_{n}(X, Y)$ with the set of triples $(Z, U, g)$ where $Z$ is a closed subset of $X \times\left(\mathbb{P}^{1}\right)^{\times n}$ that does not intersect the complement of $\mathbb{A}_{X}^{n}$. Thus $Z$ is simultaneously affine and projective over $X$, hence finite. Writing the map $g: U \rightarrow \mathbb{A}_{Y}^{n}$ as a pair $g=(\phi, f), \phi: U \rightarrow$ $\mathbb{A}_{k}^{n}, f: U \rightarrow Y$, we get the following description: the set $\operatorname{Fr}_{n}(X, Y)$ equals to the set of equivalence classes of quadruples $(Z, U, \phi, f)$ where

(1) $Z$ is a closed subset of $\mathbb{A}_{X}^{n}$ finite over $X$, 
(2) $U$ is an étale neighborhood of $Z$ in $\mathbb{A}_{X}^{n}$,

(3) $\phi: U \rightarrow \mathbb{A}_{k}^{n}$ is a regular morphism such that $\phi^{-1}(0)=Z$,

(4) $f: U \rightarrow Y$ is a regular morphism.

The quadruples $(Z, U, \phi, f)$ and $\left(Z^{\prime}, U^{\prime}, \phi^{\prime}, f^{\prime}\right)$ are equivalent if $Z=Z^{\prime}$ and there is an étale neighborhood $V$ of $Z$ in $U \times_{X} U^{\prime}$ such that $\phi$ and $f$ coincide on $V$ with $\phi^{\prime}$ and $f^{\prime}$ respectively.

2.5. Definition ([9, Definition 2.3]). The category $\mathrm{Fr}_{+}(k)$ has the objects those of $\mathrm{Sm}_{k}$, the hom sets given by

$$
\operatorname{Hom}_{\mathrm{Fr}_{+}(k)}(X, Y)=\bigvee_{n \geqslant 0} \operatorname{Fr}_{n}(X, Y)
$$

and the composition given by Definition 2.3. There is an obvious functor $\mathrm{Sm}_{k} \rightarrow \mathrm{Fr}_{+}(k)$ identical on objects and tautological on the morphisms

$$
\operatorname{Hom}_{\mathrm{Sm}_{k}}(X, Y) \rightarrow \operatorname{Hom}_{\mathrm{Shv}}\left(X_{+}, Y_{+}\right)=\operatorname{Fr}_{0}(X, Y) .
$$

A presheaf on the category $\operatorname{Fr}_{+}(k)$ is called a framed presheaf. For $X \in \operatorname{Sm}_{k}$ we denote $\operatorname{Fr}_{+}(X)$ the framed presheaf given by $U \mapsto \mathrm{Fr}_{+}(U, X)$.

2.6. Definition ([9, Definition 2.4]). For $F, F^{\prime}, G, G^{\prime} \in \operatorname{Shv} \bullet$ and $a \in \operatorname{Fr}_{n}(F, G), b \in \operatorname{Fr}_{m}\left(F^{\prime}, G^{\prime}\right)$ the external product $a \bigotimes b \in \operatorname{Fr}_{n+m}\left(F \wedge F^{\prime}, G \wedge G^{\prime}\right)$ is given by the composition

$$
F \wedge F^{\prime} \wedge \mathbb{P}^{\wedge n} \wedge \mathbb{P}^{\wedge m} \stackrel{t w_{1}}{\rightarrow} F \wedge \mathbb{P}^{\wedge n} \wedge F^{\prime} \wedge \mathbb{P}^{\wedge m} \stackrel{a \wedge b}{\rightarrow} G \wedge T^{n} \wedge G^{\prime} \wedge T^{m} \stackrel{t w_{2}}{\rightarrow} G \wedge G^{\prime} \wedge T^{n} \wedge T^{m}
$$

where $t w_{1}$ twists $\mathbb{P}^{\wedge n}$ with $F^{\prime}$ and $t w_{2}$ twists $T^{n}$ with $G^{\prime}$.

2.7. Definition. The external product with $\sigma: \mathbb{P}^{1} \rightarrow T$ considered as an element of $\operatorname{Fr}_{1}(k, k)$ gives rise to the suspension map

$$
\Sigma: \operatorname{Fr}_{n}(F, G) \rightarrow \operatorname{Fr}_{n+1}(F, G), \quad a \mapsto a \otimes \sigma .
$$

For $F \in \operatorname{Shv} \bullet$ denote $\sigma_{F}$ the element $\Sigma\left(\operatorname{id}_{F}\right) \in \operatorname{Fr}_{1}(F, F)$. Then for $a \in \operatorname{Fr}_{n}(F, G)$ one has

$$
\Sigma(a)=\sigma_{G} \circ a .
$$

2.8. Definition ([9, Definition 8.3], [10, $\S 2])$. For $X, Y \in \mathrm{Sm}_{k}$ put

$$
\mathbb{Z} \mathrm{F}_{n}(X, Y)=\mathbb{Z}\left[\operatorname{Fr}_{n}(X, Y)\right] / A
$$

with $A$ being the subgroup generated by the formal linear combinations

$$
\left(Z_{1} \sqcup Z_{2}, U, \phi, f\right)-\left(Z_{1}, U-Z_{2}, \phi, f\right)-\left(Z_{2}, U-Z_{1}, \phi, f\right) .
$$

The category $\mathbb{Z F}_{*}(k)$ has the objects those of $\mathrm{Sm}_{k}$, hom sets given by

$$
\operatorname{Hom}_{\mathbb{Z} F_{*}(k)}(X, Y)=\bigoplus_{n \geqslant 0} \mathbb{Z F}_{n}(X, Y)
$$

and the composition induced by the composition in $\operatorname{Fr}_{+}(k)$. Note that $\mathbb{Z F}_{*}(k)$ is an additive category with the direct sum given by $X \sqcup Y$ and that there are obvious functors $\mathrm{Sm}_{k} \rightarrow$ $\mathrm{Fr}_{+}(k) \rightarrow \mathbb{Z} \mathrm{F}_{*}(k)$. An additive contravariant functor $\mathbb{Z} \mathrm{F}_{*}(k) \rightarrow \mathrm{Ab}$ is called a $\mathbb{Z} \mathrm{F}_{*}$-presheaf (or a linear framed presheaf). A $\mathbb{Z} \mathrm{F}_{*}$-presheaf restricting to a sheaf on $\mathrm{Sm}_{k}$ is called a $\mathbb{Z} \mathrm{F}_{*}$-sheaf. We say that a presheaf of abelian groups $F: \mathrm{Sm}_{k}^{o p} \rightarrow \mathrm{Ab}$ admits a structure of a $\mathbb{Z F} \mathrm{F}_{*}$-presheaf if it can be extended to a $\mathbb{Z} \mathrm{F}_{*}$-presheaf,

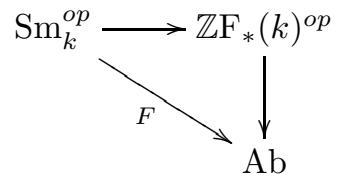


2.9. Definition. For $X \in \operatorname{Sm}_{k}$ denote $\mathbb{Z F}_{n}(X)$ the presheaf given by

$$
U \mapsto \mathbb{Z} \mathrm{F}_{n}(U, X)
$$

The suspension map of Definition 2.7 descends to a morphism of presheaves

$$
\Sigma: \mathbb{Z F}_{n}(X) \rightarrow \mathbb{Z F}_{n+1}(X)
$$

and gives rise to a $\mathbb{Z} F_{*}$-presheaf

$$
\mathbb{Z} \mathrm{F}(X)=\operatorname{colim}\left(\mathbb{Z F}_{0}(X) \stackrel{\Sigma}{\rightarrow} \mathbb{Z}_{1}(X) \stackrel{\Sigma}{\rightarrow} \ldots \stackrel{\Sigma}{\rightarrow} \mathbb{Z}_{n}(X) \stackrel{\Sigma}{\rightarrow} \ldots\right) .
$$

2.10. Lemma (cf. [10, the discussion above Theorem 1.1]). A framed presheaf of abelian groups $F$ admits a structure of a $\mathbb{Z F}_{*}$-presheaf if and only if the natural map $F(X \sqcup Y) \rightarrow F(X) \oplus F(Y)$ is an isomorphism for all $X, Y \in \mathrm{Sm}_{k}$.

Proof. A $\mathbb{Z F}_{*}$-presheaf is an additive functor, so one implication is clear.

Suppose that $F$ is a framed presheaf and $F(X \sqcup Y) \rightarrow F(X) \oplus F(Y)$ is an isomorphism for every $X, Y \in \operatorname{Sm}_{k}$. For $Y \in \operatorname{Sm}_{k}$ let

$$
p, p_{1}, p_{2} \in \operatorname{Fr}_{0}(Y \sqcup Y, Y)=\operatorname{Hom}_{\mathrm{Shv}}\left(Y_{+} \vee Y_{+}, Y_{+}\right)
$$

be the following morphisms:

- $p$ is the identity on both copies of $Y$,

- $p_{1}$ is the identity on the first copy of $Y$ and sends the second copy of $Y$ to the distinguished point,

- $p_{2}$ is the identity on the second copy of $Y$ and sends the first copy of $Y$ to the distinguished point.

By the assumption we have

$$
p^{*}=p_{1}^{*}+p_{2}^{*}: F(Y) \rightarrow F(Y \sqcup Y) .
$$

Consider $c=\left(Z_{1} \sqcup Z_{2}, U, \phi, f\right) \in \operatorname{Fr}_{n}(X, Y)$. Then for

$$
c^{\prime}=\left(Z_{1} \sqcup Z_{2},\left(U-Z_{2}\right) \sqcup\left(U-Z_{1}\right), \phi \sqcup \phi, f \sqcup f\right) \in \operatorname{Fr}_{n}(X, Y \sqcup Y)
$$

we have

$$
p \circ c^{\prime}=\left(Z_{1} \sqcup Z_{2}, U, \phi, f\right), \quad p_{1} \circ c^{\prime}=\left(Z_{1}, U-Z_{2}, \phi, f\right), \quad p_{2} \circ c^{\prime}=\left(Z_{2}, U-Z_{1}, \phi, f\right)
$$

in $\operatorname{Fr}_{n}(X, Y)$. Put $c_{1}=p_{1} \circ c^{\prime}, c_{2}=p_{2} \circ c^{\prime}$. By the above we have

$$
c^{*}=\left(c^{\prime}\right)^{*} \circ p^{*}=\left(c^{\prime}\right)^{*} \circ\left(p_{1}^{*}+p_{2}^{*}\right)=c_{1}^{*}+c_{2}^{*},
$$

thus $F$ descends to a functor on $\mathbb{Z F}_{*}(k)$.

2.11. Definition $([10, \S 1])$. A $\mathbb{Z} F_{*}$-presheaf $M$ is called

- stable if $\sigma_{X}^{*}: M(X) \rightarrow M(X)$ equals to the identity map on $M(X)$,

- homotopy invariant if for every $X \in \operatorname{Sm}_{k}$ the projection $p: \mathbb{A}^{1} \times X \rightarrow X$ induces an isomorphism $p^{*}: M(X) \rightarrow M\left(X \times \mathbb{A}^{1}\right)$.

2.12. Definition. For $X \in \operatorname{Sm}_{k}$ denote $\mathrm{H}_{0}^{\mathbb{Z F}}(X)$ the sheaf associated with the presheaf

$$
U \mapsto \operatorname{coker}\left[\mathbb{Z F}\left(U \times \mathbb{A}^{1}, X\right) \stackrel{i_{0}^{*}-i_{1}^{*}}{\longrightarrow} \mathbb{Z} \mathrm{F}(U, X)\right] .
$$

Here $i_{0}, i_{1}: U \rightarrow U \times \mathbb{A}^{1}$ are given by $i_{0}(u)=(u, 0)$ and $i_{1}(u)=(u, 1)$ respectively. For $n \geqslant 0$ put

$$
\mathrm{H}_{0}^{\mathbb{Z F}}\left(X \times \mathbb{G}_{m}^{\wedge n}\right)=\text { coker }\left[\bigoplus_{i=1}^{n} \mathrm{H}_{0}^{\mathbb{Z F}}\left(X \times\left(\mathbb{G}_{m}\right)^{\times(n-1)}\right) \stackrel{\sum f_{i}}{\longrightarrow} \mathrm{H}_{0}^{\mathbb{Z F}}\left(X \times\left(\mathbb{G}_{m}\right)^{\times n}\right)\right]
$$


with $f_{i}$ induced by the embedding $\mathbb{G}_{m}^{n-1} \rightarrow \mathbb{G}_{m}^{n}$ inserting 1 at the $i$-th place.

2.13. Lemma. For $X \in \operatorname{Sm}_{k}$ the sheaf $\mathrm{H}_{0}^{\mathbb{Z F}}(X)$ admits a canonical structure of a homotopy invariant stable $\mathbb{Z} \mathrm{F}_{*}$-presheaf.

Proof. The presheaf

$$
U \mapsto \operatorname{coker}\left[\mathbb{Z F}\left(U \times \mathbb{A}^{1}, X\right) \stackrel{i_{0}^{*}-i_{1}^{*}}{\longrightarrow} \mathbb{Z} \mathrm{F}(U, X)\right]
$$

admits a canonical structure of a $\mathbb{Z F}_{*}$-presheaf being the cokernel of a morphism of $\mathbb{Z F}_{*^{-}}$ presheaves. It is immediate to see that this presheaf is homotopy invariant and [15, the proof of Remark 2.3] yields that it is stable. Thus [10, Theorem 2.1] gives the claim.

2.14. Lemma. Let $M$ be a homotopy invariant stable $\mathbb{Z F}_{*}$-sheaf. Then for $X \in \operatorname{Sm}_{k}$ and $p \geqslant 0$ the canonical morphism

is an isomorphism.

$$
H_{Z a r}^{p}(X, M) \rightarrow H_{N i s}^{p}(X, M)
$$

Proof. Consider the Leray spectral sequence

$$
H_{Z a r}^{p}\left(X, \mathcal{H}_{N i s}^{q}(M)\right) \Rightarrow H_{N i s}^{p+q}(X, M)
$$

where $\mathcal{H}_{N i s}^{q}(M)$ is the Zariski sheafification of the presheaf $U \mapsto H_{N i s}^{q}(X, M)$. The homomorphism

$$
H_{N i s}^{q}\left(\operatorname{Spec} \mathcal{O}_{X, x}, M\right) \rightarrow H_{N i s}^{q}(\operatorname{Spec} k(X), M)
$$

is injective by [10, Theorems 2.15(3), 16.10 and 16.11]. Thus for $q>0$ we have

$$
H_{N i s}^{q}\left(\operatorname{Spec} \mathcal{O}_{X, x}, M\right)=0
$$

whence $\mathcal{H}_{N i s}^{q}(M)=0$. The spectral sequence degenerates yielding the claim.

2.15. Definition. For a presheaf $M$ let $M_{-1}$ be the presheaf given by

$$
X \mapsto \operatorname{ker}\left[M\left(\mathbb{G}_{m} \times X\right) \stackrel{i_{1}^{*}}{\rightarrow} M(X)\right]
$$

for the embedding $i_{1}: X \rightarrow \mathbb{G}_{m} \times X, i_{1}(x)=(1, x)$. It is straightforward to see that if $M$ is a homotopy invariant stable $\mathbb{Z} \mathrm{F}_{*}$-sheaf then $M_{-1}$ is also homotopy invariant stable $\mathbb{Z} \mathrm{F}_{*}$-sheaf.

2.16. Lemma. The functor $M \mapsto M_{-1}$ is exact on the category of homotopy invariant stable $\mathbb{Z F}_{*}$-sheaves.

Proof. It is clear that the functor is left exact.

Suppose that $M \rightarrow N$ is an epimorphism of homotopy invariant stable $\mathbb{Z F}_{*}$-sheaves. The projection $p: \mathbb{G}_{m} \times X \rightarrow X$ gives a splitting

$$
M\left(\mathbb{G}_{m} \times X\right) \cong M_{-1}(X) \oplus M(X)
$$

functorial in $X$, thus it is sufficient to show that $M\left(\mathbb{G}_{m} \times U\right) \rightarrow N\left(\mathbb{G}_{m} \times U\right)$ is surjective for a local $U$.

The cokernel presheaf $Q=\operatorname{coker}(M \rightarrow N)$ is a homotopy invariant stable $\mathbb{Z F}_{*}$-presheaf as well as the presheaf $Q\left(\mathbb{G}_{m} \times-\right)$. By [10, Theorem 2.15(3)] the pullback

$$
Q\left(\mathbb{G}_{m} \times U\right) \rightarrow Q\left(\mathbb{G}_{m} \times \operatorname{Spec} k(U)\right)
$$

is injective. Applying [10, Theorem 2.15(1)] to the presheaf $Q$ considered as a $\mathbb{Z} \mathrm{F}_{*}(k(U))$-presheaf we obtain that the pullback

$$
Q\left(\mathbb{G}_{m} \times k(U)\right) \rightarrow \underset{6}{Q\left(\operatorname{Spec} k\left(\mathbb{G}_{m} \times U\right)\right)}
$$


is injective whence the pullback

$$
Q\left(\mathbb{G}_{m} \times U\right) \rightarrow Q\left(\operatorname{Spec} k\left(\mathbb{G}_{m} \times U\right)\right)
$$

is injective as well. By the assumption $Q\left(\operatorname{Spec} k\left(\mathbb{G}_{m} \times U\right)\right)=0$ whence the claim.

\section{Framed Structure For homotopy modules}

3.1. Definition. Let $\Pi_{*}(k)$ denote the category of homotopy modules, i.e. pairs $\left(M_{*}, \phi_{*}\right)$ where $M_{*}$ is a $\mathbb{Z}$-graded strictly homotopy invariant sheaf and $\phi_{i}: M_{i} \rightarrow\left(M_{i+1}\right)_{-1}$ are isomorphisms. We usually shorten the notation and refer to a homotopy module $\left(M_{*}, \phi_{*}\right)$ as $M_{*}$.

3.2. Remark. By [14, Theorem 5.2.6] there is a pair of functors

$$
\pi_{0}(-)_{*}: \mathrm{SH}(k) \leftrightarrows \Pi_{*}(k): H
$$

that provides an equivalence between $\Pi_{*}(k)$ and the heart of the homotopy t-structure on $\mathrm{SH}(k)$. Here $\pi_{0}(E)_{*}$ is the graded sheaf associated to the family of presheaves

$$
\left\{U \mapsto \operatorname{Hom}_{\mathrm{SH}(k)}\left(\Sigma_{\mathbb{P}^{1}}^{\infty} U_{+}, E \wedge \mathbb{G}_{m}^{\wedge i}\right)\right\}_{i \in \mathbb{Z}}
$$

and $H M_{*}$ is the Eilenberg-Maclane spectrum of the homotopy module $M_{*}$ [14, the discussion above Theorem 5.2.6]. The main result of this section claims that every homotopy module $M_{*}$ carries a unique structure of a homotopy invariant stable $\mathbb{Z F}_{*}$-sheaf such that the isomorphisms $\phi_{i}: M_{i} \rightarrow\left(M_{i+1}\right)_{-1}$ are compatible with the framed transfers.

3.3. Definition. For $X, Y \in \mathrm{Sm}_{k}$ a framed correspondence

$$
a \in \operatorname{Fr}_{n}(X, Y)=\operatorname{Hom}_{\text {Shv }}\left(X_{+} \wedge \mathbb{P}^{\wedge n}, Y_{+} \wedge T^{n}\right)
$$

gives rise to a morphism

$$
a_{*}=\Sigma_{\mathbb{P}^{1}}^{-n}\left(\left(\Sigma_{\mathbb{P}^{1}}^{\infty}\left(\operatorname{id}_{Y_{+}} \wedge \sigma^{n}\right)\right)^{-1} \circ \Sigma_{\mathbb{P}^{1}}^{\infty} a\right): \Sigma_{\mathbb{P}^{1}}^{\infty} X_{+} \rightarrow \Sigma_{\mathbb{P}^{1}}^{\infty} Y_{+}
$$

in the stable motivic homotopy category $\mathrm{SH}(k)$,

$$
\Sigma_{\mathbb{P}^{1}}^{\infty}\left(X_{+} \wedge \mathbb{P}^{\wedge n}\right) \stackrel{\Sigma_{\mathbb{P}^{1}}^{\infty} a}{\longrightarrow} \Sigma_{\mathbb{P}^{1}}^{\infty}\left(Y_{+} \wedge T^{n}\right)
$$

Here $\operatorname{id}_{Y_{+}} \wedge \sigma^{n}$ is the isomorphism induced by the canonical $\mathbb{A}^{1}$-equivalence $\sigma: \mathbb{P}^{1} \rightarrow T$.

3.4. Lemma. The assignment $X \mapsto \Sigma_{\mathbb{P 1}}^{\infty} X_{+}, a \mapsto a_{*}$ defines a functor

$$
r: \mathbb{Z} \mathrm{F}_{*}(k) \rightarrow \mathrm{SH}(k)
$$

that sends $\sigma_{X}$ to $\mathrm{id}_{\Sigma_{\mathbb{1}} X_{+}}$.

Proof. It is straightforward to see that the assignment gives rise to a functor $\tilde{r}: \operatorname{Fr}_{+}(k) \rightarrow \mathrm{SH}(k)$ with $\tilde{r}\left(\sigma_{X}\right)=\operatorname{id}_{\Sigma_{\mathbb{p} 1}^{\infty} X_{+}}$. Moreover, $\tilde{r}$ satisfies

$$
\tilde{r}(X \sqcup Y)=\tilde{r}(X) \oplus \tilde{r}(Y)
$$

for all $X, Y \in \mathrm{Sm}_{k}$. The claim follows by the same reasoning as in Lemma 2.10. 
3.5. Definition. Let $\Pi_{*}^{\mathrm{fr}}(k)$ denote the category of framed homotopy modules, i.e. the category of pairs $\left(M_{*}, \phi_{*}\right)$ where $M_{*}$ is a $\mathbb{Z}$-graded homotopy invariant stable $\mathbb{Z F}_{*}$-sheaf and $\phi_{i}: M_{i} \rightarrow$ $\left(M_{i+1}\right)_{-1}$ are isomorphisms of $\mathbb{Z F}_{*}$-sheaves. We usually shorten the notation and refer to a framed homotopy module $\left(M_{*}, \phi_{*}\right)$ as $M_{*}$. Recall that a homotopy invariant stable $\mathbb{Z F}_{*}$-sheaf is strictly homotopy invariant by [10, Theorems 16.10 and 16.11]. Forgetting about the framed structure we obtain a forgetful functor

$$
\Phi^{\mathrm{fr}}: \Pi_{*}^{\mathrm{fr}}(k) \rightarrow \Pi_{*}(k) .
$$

3.6. Lemma. The functor $\pi_{0}(-)_{*}: \mathrm{SH}(k) \rightarrow \Pi_{*}(k)$ factors through the category $\Pi_{*}^{\mathrm{fr}}(k)$ making the following diagram commute.

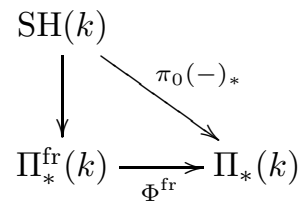

Proof. Lemma 3.4 yields that for $E \in \mathrm{SH}(k)$ and every $i \in \mathbb{Z}$ the presheaf

$$
U \mapsto \operatorname{Hom}_{\mathrm{SH}(k)}\left(\Sigma_{\mathbb{P}^{1}}^{\infty} U_{+}, E \wedge \mathbb{G}_{m}^{\wedge i}\right)
$$

is a stable $\mathbb{Z F}_{*}$-presheaf which is obviously homotopy invariant. The associated sheaves $\pi_{0}(E)_{i}$ are homotopy invariant stable $\mathbb{Z F}_{*}$-sheaves by [10, Theorem 2.1]. For every morphism $E \rightarrow E^{\prime}$ in $\mathrm{SH}(k)$ the induced map $\pi_{0}(E)_{i} \rightarrow \pi_{0}\left(E^{\prime}\right)_{i}$ is a morphism of $\mathbb{Z F}_{*}$-sheaves, thus the isomorphisms $\pi_{0}(E)_{i} \rightarrow\left(\pi_{0}(E)_{i+1}\right)_{-1}$ associated to the isomorphisms $E \rightarrow \underline{\operatorname{Hom}}\left(\Sigma_{\mathbb{P}^{1}}^{\infty} \mathbb{G}_{m}^{\wedge 1}, E \wedge \mathbb{G}_{m}^{\wedge 1}\right)$ respect framed structure.

3.7. Definition. Let $\pi_{0}^{\mathrm{fr}}(-)_{*}: \mathrm{SH}(k) \rightarrow \Pi_{*}^{\mathrm{fr}}(k)$ be the functor given by Lemma 3.6. Then the composition with the Eilenberg-Maclane functor gives rise to a functor

$$
\Psi^{\mathrm{fr}}=\pi_{0}^{\mathrm{fr}}(-)_{*} \circ H: \Pi_{*}(k) \rightarrow \Pi_{*}^{\mathrm{fr}}(k) .
$$

3.8. Definition. For $X \in \operatorname{Sm}_{k}$ let $\mathrm{H}_{0}^{\mathbb{Z F}}(X)_{*}$ be the $\mathbb{Z}$-graded $\mathbb{Z} \mathrm{F}_{*}$-sheaf given by

$$
\mathrm{H}_{0}^{\mathbb{Z F}}(X)_{i}=\left[\begin{array}{ll}
\mathrm{H}_{0}^{\mathbb{Z F}}\left(X \times \mathbb{G}_{m}^{\wedge i}\right), & i \geqslant 0 \\
\left(\mathrm{H}_{0}^{\mathbb{Z F}}(X)\right)_{-|i|}, & i<0 .
\end{array}\right.
$$

Then $\mathrm{H}_{0}^{\mathbb{Z}} \mathrm{F}(X)_{*}$ is a framed homotopy module by [2, Theorem $\left.\mathrm{C}\right]$.

3.9. Lemma. There is a canonical isomorphism of framed homotopy modules

$$
\mathrm{H}_{0}^{\mathbb{Z F}}(X)_{*} \cong \Psi^{\mathrm{fr}} \Phi^{\mathrm{fr}} \mathrm{H}_{0}^{\mathbb{Z F}}(X)_{*} .
$$

Proof. For every $i \in \mathbb{Z}$ the functor $\mathbb{Z F}_{*}(k) \rightarrow \mathrm{SH}(k)$ of Lemma 3.4 gives rise to a morphism of $\mathbb{Z} \mathrm{F}_{*}$-presheaves

$$
\mathbb{Z F}_{*}\left(X \times \mathbb{G}_{m}^{\wedge i}\right) \rightarrow \operatorname{Hom}_{\mathrm{SH}(k)}\left(-, \Sigma_{\mathbb{P}^{1}}^{\infty} X_{+} \wedge \mathbb{G}_{m}^{\wedge i}\right)
$$

that descends to an isomorphism of framed sheaves

$$
\mathrm{H}_{0}^{\mathbb{Z F}}(X)_{i} \stackrel{\simeq}{\rightarrow} \pi_{0}^{\mathrm{fr}}\left(\Sigma_{\mathbb{P}^{1}}^{\infty} X_{+}\right)_{i}
$$

by [9, Theorem 11.1 and Corollary 11.3]. The canonical map

$$
\Sigma_{\mathbb{P} 1}^{\infty} X_{+} \rightarrow H \pi_{0}\left(\Sigma_{\mathbb{P} 1}^{\infty} X_{+}\right)_{*}
$$

induces an isomorphism

$$
\pi_{0}^{\mathrm{fr}}\left(\Sigma_{\mathbb{P}^{1}}^{\infty} X_{+}\right)_{i} \rightarrow \pi_{0}^{\mathrm{fr}}\left(H \pi_{0}\left(\Sigma_{\mathbb{P}^{1}}^{\infty} X_{+}\right)_{*}\right)_{i}
$$

whence

$$
\mathrm{H}_{0}^{\mathbb{Z F}}(X)_{i} \cong \pi_{0}^{\mathrm{fr}}\left(\Sigma_{\mathbb{P}^{1}}^{\infty} X_{+}\right)_{i} \cong \Psi_{8}^{\mathrm{fr}} \Phi_{0}^{\mathrm{fr}} \pi_{0}^{\mathrm{fr}}\left(\Sigma_{\mathbb{P}^{1}}^{\infty} X_{+}\right)_{i} \cong \Psi^{\mathrm{fr}} \Phi^{\mathrm{fr}} \mathrm{H}_{0}^{\mathbb{Z F}}(X)_{i}
$$


3.10. Lemma. For a framed homotopy module $M_{*}$ there exists is a surjection of framed homotopy modules $\bigoplus_{j \in J} \mathrm{H}_{0}^{\mathbb{Z F}}\left(X_{j}\right)_{*+n_{j}} \rightarrow M_{*}$ for some $J, X_{j}$ and $n_{j}$.

Proof. Every $a \in M_{i}(X)$ defines a morphism of $\mathbb{Z F}_{*}$-presheaves $\mathbb{Z F}_{*}(X) \rightarrow M_{i}$. Since $M_{i}$ is homotopy invariant and stable, the morphism factors through $\mathrm{H}_{0}^{\mathbb{Z F}}(X)$ yielding a morphism $\rho_{a}: \mathrm{H}_{0}^{\mathbb{Z F}}(X) \rightarrow M_{i}$. The isomorphism

$$
\phi_{i}: M_{i}(X) \cong\left(M_{i+1}\right)_{-1}(X)=M_{i+1}\left(X \times \mathbb{G}_{m}^{\wedge 1}\right)
$$

combined with the reasoning above gives rise to a morphism of $\mathbb{Z} \mathrm{F}_{*}$-sheaves

$$
\rho_{\phi_{i}(a)}: \mathrm{H}_{0}^{\mathbb{Z F}}(X)_{1} \rightarrow M_{i+1} .
$$

Iterating we obtain compatible morphisms of $\mathbb{Z F}_{*}$-sheaves

$$
\mathrm{H}_{0}^{\mathbb{Z F}}(X)_{m} \rightarrow M_{i+m},
$$

i.e. a morphism of framed homotopy modules $\mathrm{H}_{0}^{\mathbb{Z F}}(X)_{*-i} \rightarrow M_{*}$. The direct sum of such morphisms for all $a \in M_{*}(X)$ and all (isomorphism classes) of $X$ gives the claim.

3.11. Proposition. The functors

$$
\Phi^{\mathrm{fr}}: \Pi_{*}^{\mathrm{fr}}(k) \rightleftarrows \Pi_{*}(k): \Psi^{\mathrm{fr}}
$$

are inverse equivalences of categories.

Proof. The isomorphism $\Phi^{\mathrm{fr}} \circ \Psi^{\mathrm{fr}} \cong \mathrm{id}$ is immediate.

For a framed homotopy module $M_{*}$ Lemma 3.10 provides a resolution

$$
\ldots \rightarrow \bigoplus_{j \in J_{2}} \mathrm{H}_{0}^{\mathbb{Z F}}\left(X_{j}\right)_{*+n_{j}} \rightarrow \bigoplus_{j \in J_{1}} \mathrm{H}_{0}^{\mathbb{Z F}}\left(X_{j}\right)_{*+n_{j}} \rightarrow \bigoplus_{j \in J_{0}} \mathrm{H}_{0}^{\mathbb{Z F}}\left(X_{j}\right)_{*+n_{j}} \rightarrow M_{*} .
$$

Lemma 3.9 shows that $\Psi^{\text {fr }} \circ \Phi^{\text {fr }} \cong$ id for the resolution whence the claim.

3.12. Definition. Let $M_{*}$ be a homotopy module. For a sheaf of abelian groups $F$ put

$$
\operatorname{Ext}^{n}\left(F, M_{*}\right)=\operatorname{Hom}_{\mathrm{D}(\operatorname{ShvAb})}\left(F, M_{*}[n]\right)
$$

with $M_{*}=\bigoplus_{i \in \mathbb{Z}} M_{i}$ considered as a complex concentrated in the zeroth degree. Then for a closed subset $S \subset X \in \mathrm{Sm}_{k}$ we have the following isomorphisms:

$$
\begin{aligned}
H_{S}^{i}\left(X, M_{*}\right) \cong H_{S \times \mathbb{G}_{m}^{\wedge n}}^{i} & \left(X \times \mathbb{G}_{m}^{\wedge n}, M_{*+n}\right) \cong \\
\cong & \operatorname{Hom}_{\mathrm{D}(\operatorname{ShvAb})}\left(\mathbb{Z}\left[(X / X-S) \wedge \mathbb{G}_{m}^{\wedge n}\right], M_{*+n}[i]\right) \cong \\
& \cong \operatorname{Ext}^{n+i}\left(\mathbb{Z}\left[(X / X-S) \wedge T^{n}\right], M_{*+n}\right) \cong \\
& \cong \operatorname{Ext}^{n+i}\left(\mathbb{Z}\left[(X / X-S) \wedge \mathbb{P}^{\wedge n}\right], M_{*+n}\right) .
\end{aligned}
$$

The first isomorphism is given by the proof of Lemma 2.16 combined with Proposition 3.11, the second one is well-known and the rest are induced by the canonical $\mathbb{A}^{1}$-equivalences $\mathbb{P}^{\wedge n} \sim T^{n} \sim$ $\mathbb{G}_{m}^{\wedge n} \wedge S^{n}$.

3.13. Lemma. For a homotopy module $M_{*}$ and $a \in \operatorname{Fr}_{n}(X, Y)$ the restriction $a^{*}:\left(\Psi^{\mathrm{fr}} M_{*}\right)(Y) \rightarrow$ $\left(\Psi^{\mathrm{fr}} M_{*}\right)(X)$ equals to the composition

$$
M_{*}(Y) \cong \operatorname{Ext}^{n}\left(\mathbb{Z}\left[Y \wedge T^{n}\right], M_{*+n}\right) \rightarrow \operatorname{Ext}^{n}\left(\mathbb{Z}\left[X \wedge \mathbb{P}^{\wedge n}\right], M_{*+n}\right) \cong M_{*}(X) .
$$

Proof. It is straightforward to see that the above rule endows $M_{*}$ with the structure of a framed homotopy module. The equivalence $\Psi^{\mathrm{fr}} \circ \Phi^{\mathrm{fr}} \cong$ id from Proposition 3.11 yields that such structure is unique. 
3.14. Definition. Similar to Definition 2.4, for $X, Y \in \operatorname{Sm}_{k}$ an element $a \in \operatorname{Fr}_{n}\left(X_{+}, Y_{+} \wedge T^{l}\right)$ is defined by a quadruple $(Z, U, \phi, f)$ where

(1) $Z$ is a closed subset of $\mathbb{A}_{X}^{n}$ finite over $X$,

(2) $U$ is an étale neighborhood of $Z$ in $\mathbb{A}_{X}^{n}$,

(3) $\phi: U \rightarrow \mathbb{A}_{k}^{l+n}$ is a regular morphism such that $\phi^{-1}(0)=Z$,

(4) $f: U \rightarrow Y$ is a regular morphism.

For a closed subset $S \subset Y$ put $a^{-1}(S)=\pi\left(f^{-1}(S) \cap Z\right)$ with $\pi: U \rightarrow X$ being the projection map. Then $a$ takes $\left(X-a^{-1}(S)\right)_{+} \wedge \mathbb{P}^{\wedge n}$ to $(Y-S)_{+} \wedge T^{n+l}$ and induces a morphism of sheaves

$$
\left(X / X-a^{-1}(S)\right) \wedge \mathbb{P}^{\wedge n} \rightarrow(Y / Y-S) \wedge T^{n+l} .
$$

For a homotopy module $M_{*}$ the morphism yields a homomorphism

$$
\operatorname{Ext}^{n+i}\left(\mathbb{Z}\left[(Y / Y-S) \wedge T^{n+l}\right], M_{*+n}\right) \rightarrow \operatorname{Ext}^{n+i}\left(\mathbb{Z}\left[\left(X / X-a^{-1}(S)\right) \wedge \mathbb{P}^{\wedge n}\right], M_{*+n}\right) .
$$

Applying the isomorphisms from Definition 3.12 we obtain a map

$$
a^{*}: H_{S}^{i-l}\left(Y, M_{*+n-l}\right) \rightarrow H_{a^{-1}(S)}^{i}\left(X, M_{*+n}\right) .
$$

3.15. Definition. Let $M$ be a framed presheaf. The pairing

$$
M\left(U \times \mathbb{G}_{m}^{\times n}\right) \times \mathrm{Fr}_{+}\left(U, \mathbb{G}_{m}^{\times n}\right) \rightarrow M(U)
$$

given by $(m, a) \mapsto \Delta^{*}\left(\operatorname{id}_{U} \otimes a\right)^{*}(m)$ with $\Delta: U \rightarrow U \times U$ being the diagonal map gives rise to a pairing of framed presheaves

$$
M_{-n} \times \mathrm{Fr}_{+}\left(\mathbb{G}_{m}^{\wedge n}\right) \rightarrow M .
$$

If $M$ is a homotopy invariant stable $\mathbb{Z} \mathrm{F}_{*^{-}}$-sheaf this pairing descends to a morphism of $\mathbb{Z F}_{*^{-}}$ sheaves

$$
M_{-n} \times \mathrm{H}_{0}^{\mathbb{Z F}}\left(\mathbb{G}_{m}^{\wedge n}\right) \rightarrow M
$$

Let $M_{*}$ be a homotopy module. Endow it with the framed structure via the functor $\Psi^{\text {fr }}$. In view of the identification $\mathrm{H}_{0}^{\mathbb{Z F}}\left(\mathbb{G}_{m}^{\wedge n}\right) \cong \mathrm{K}_{n}^{\mathrm{MW}}$ given by [9, Corollary 11.3] combined with [14, Theorem 6.4.1] the pairing endows $M_{*}$ with a $\mathrm{K}_{*}^{\mathrm{MW}}$-module structure $M_{*} \times \mathrm{K}_{*}^{\mathrm{MW}} \rightarrow M_{*}$ yielding a $\mathrm{K}_{*}^{\mathrm{MW}}$-module structure

$$
H_{S}^{i}\left(Y, M_{*}\right) \times \mathrm{K}_{*}^{\mathrm{MW}} \rightarrow H_{S}^{i}\left(Y, M_{*}\right)
$$

for a closed subset $S \subset Y \in \operatorname{Sm}_{k}$. Moreover, for $Y_{1}, Y_{2} \in \mathrm{Sm}_{k}$ and closed subsets $S_{1} \subset Y_{1}$, $S_{2} \subset Y_{2}$ we get external product

$$
H_{S_{1}}^{i}\left(Y_{1}, M_{*}\right) \times H_{S_{2}}^{j}\left(Y_{2}, \mathrm{~K}_{*}^{\mathrm{MW}}\right) \stackrel{\times}{\longrightarrow} H_{S_{1} \times S_{2}}^{i+j}\left(Y_{1} \times Y_{2}, M_{*}\right)
$$

induced by the external product in cohomology.

3.16. Lemma. For a framed homotopy module $M_{*}$, smooth varieties $X_{1}, Y_{1}, X_{2}, Y_{2} \in \mathrm{Sm}_{k}$, closed subsets $S_{1} \subset Y_{1}, S_{2} \subset Y_{2}$ and $a_{1} \in \mathrm{Fr}_{+}\left(X_{1}, Y_{1}\right), a_{2} \in \mathrm{Fr}_{+}\left(X_{2}, Y_{2}\right)$ the following diagram commutes.

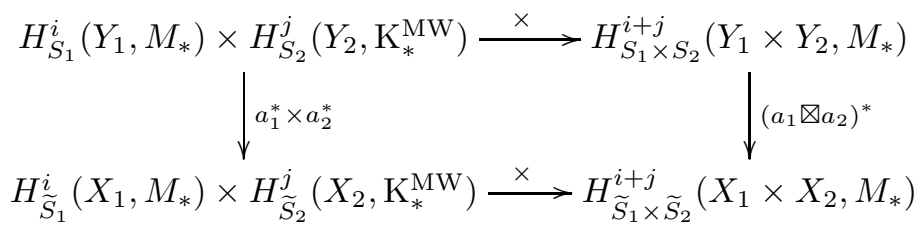

Here $\widetilde{S}_{1}=a_{1}^{-1}\left(S_{1}\right), \widetilde{S}_{2}=a_{2}^{-1}\left(S_{2}\right)$ in the notation of Definition 3.14.

Proof. Straightforward, since all the maps $a_{1}^{*}, a_{2}^{*}$ and $\left(a_{1} \otimes a_{2}\right)^{*}$ are induced by the respective morphisms of sheaves. 
3.17. Lemma. Let $M_{*}$ be a homotopy module, $S \subset Y \in \operatorname{Sm}_{k}$ be a closed subset and $a \in \operatorname{Fr}_{n}(Y, k)$. Then

$$
\left(\left(\operatorname{id}_{Y} \otimes a\right) \circ \Delta_{Y}\right)^{*}(\alpha)=\alpha \cdot \bar{a}
$$

for every $\alpha \in H_{S}^{i}\left(Y, M_{*}\right)$. Here

- $\Delta_{Y}: Y \rightarrow Y \times Y$ is the diagonal morphism,

- $\left(\left(\mathrm{id}_{Y} \otimes a\right) \circ \Delta_{Y}\right)^{*}$ is given by Definition 3.14,

- $\bar{a} \in \mathrm{K}_{0}^{\mathrm{MW}}(Y)$ is the image of a under the composition

$$
\operatorname{Fr}_{n}(Y, k) \rightarrow \mathrm{H}_{0}^{\mathbb{Z F}}(k)(Y) \cong \mathrm{K}_{0}^{\mathrm{MW}}(Y)
$$

- $\alpha \cdot \bar{a}$ is given by the module structure of Definition 3.15 .

Proof. Both sides of the equality are given by the composition $\Xi=\alpha \circ\left(\operatorname{id}_{Y / Y-S} \wedge a\right) \circ\left(\Delta_{Y} \wedge\right.$ id $)$,

$$
\Xi:(Y / Y-S) \wedge \mathbb{P}^{\wedge n} \rightarrow(Y / Y-S) \wedge Y_{+} \wedge \mathbb{P}^{\wedge n} \rightarrow(Y / Y-S) \wedge T^{n} \rightarrow M_{*+n}[n+i] .
$$

3.18. Lemma. For $Y \in \operatorname{Sm}_{k}$ and $A \in \mathrm{GL}_{n}(Y)$ consider the correspondence

$$
c_{A}=\left(Y \times\{0\}, \mathbb{A}_{Y}^{n},\left(x_{1}, \ldots, x_{n}\right) \cdot A, \pi_{Y}\right) \in \operatorname{Fr}_{n}(Y, Y)
$$

with $x_{i}$ being the coordinates on $\mathbb{A}^{n}$ and $\pi_{Y}: \mathbb{A}_{Y}^{n} \rightarrow Y$ being the projection. Then for a framed homotopy module $M_{*}$ and a closed subset $S \subset Y$ the induced map

$$
c_{A}^{*}: H_{S}^{i}\left(Y, M_{*}\right) \rightarrow H_{S}^{i}\left(Y, M_{*}\right)
$$

coincides with the multiplication by $\langle\operatorname{det}(A)\rangle \in \underline{\mathrm{GW}}(Y)$.

Proof. We have

$$
c_{A}=\left(\mathrm{id}_{Y} \otimes a\right) \circ \Delta_{Y}
$$

for $a=\left(Y \times\{0\}, \mathbb{A}_{Y}^{n},\left(x_{1}, \ldots, x_{n}\right) \cdot A, \pi\right) \in \operatorname{Fr}_{n}(Y, k)$ with $\pi: \mathbb{A}_{Y}^{n} \rightarrow$ Spec $k$ being the projection. Lemma 3.17 yields that $c_{A}^{*}$ coincides with the multiplication by the image of $a$ in

$$
\mathrm{H}_{0}^{\mathbb{Z F}}(k)(Y) \cong \mathrm{K}_{0}^{\mathrm{MW}}(Y)=\underline{\mathrm{GW}}(Y) .
$$

Over a local ring every matrix of determinant 1 is elementary [17, III, Lemma 1.4], whence for every $y \in Y$ we have $A=\left((\operatorname{det} A) \oplus I_{n-1}\right) \cdot B_{y}$ for the unit matrix $I_{n-1}$ and some elementary matrix $B_{y} \in \operatorname{GL}\left(\mathcal{O}_{Y, y}\right)$. Since $B_{y}$ is $\mathbb{A}^{1}$-homotopy equivalent to the identity matrix then the image of $a$ in $\underline{\mathrm{GW}}(Y)$ coincides with $\langle\operatorname{det}(A)\rangle$.

\section{Framing as a trivialization of the Conormal Sheaf}

4.1. Definition. For $X, Y \in \operatorname{Sm}_{k}$ let $\operatorname{Fr}_{n}^{\mathcal{V}}\left(X, Y \wedge T^{l}\right)$ be the set of triples $c=(Z, \phi, g)$ where

- $Z$ is a closed (not necessarily reduced) subscheme of $\mathbb{A}_{X}^{n}$ finite over $X$,

- $\phi=\left(\phi_{1}, \ldots, \phi_{n+l}\right), \phi_{i} \in H^{0}\left(Z, I / I^{2}\right)$, is a collection of global sections that generate $I / I^{2}$ as an $\mathcal{O}_{Z}$-module with $I$ being the sheaf of ideals defining $Z$,

- $g: Z \rightarrow Y$ is a regular map.

We refer to $Z^{\text {red }}$ as the support of $c$. For $c=(Z, \phi, g) \in \operatorname{Fr}_{n}^{\mathcal{V}}\left(X, Y \wedge T^{l}\right)$ put

$$
\Sigma(c)=(Z \times 0, \Sigma(\phi), g) \in \operatorname{Fr}_{n+1}^{\mathcal{V}}\left(X, Y \wedge T^{l}\right),
$$

where $Z \times 0$ is the closed subset in $\mathbb{A}_{X}^{n+1}=\mathbb{A}_{X}^{n} \times \mathbb{A}^{1}$ and $\Sigma(\phi)=\left(\phi_{1}, \ldots, \phi_{n+l}, t\right)$ with $t$ being the coordinate function on the last copy of $\mathbb{A}^{1}$.

4.2. Definition. For $X, Y \in \operatorname{Sm}_{k}$ take $\left(Z, U,\left(\phi_{1}, \ldots, \phi_{n+l}\right), g\right) \in \operatorname{Fr}_{n}\left(X_{+}, Y_{+} \wedge T^{l}\right)$. Here

(1) $Z$ is a closed subset of $\mathbb{A}_{X}^{n}$ finite over $X$,

(2) $U$ is an étale neighborhood of $Z$ in $\mathbb{A}_{X}^{n}$,

(3) $\phi=\left(\phi_{1}, \ldots, \phi_{n+l}\right): U \rightarrow \mathbb{A}_{k}^{n+l}$ such that $\phi^{-1}(0)=Z$, 
(4) $g: U \rightarrow Y$ is a regular morphism.

Let $\widetilde{Z}=U \times_{\mathbb{A}_{k}^{n+l}}$ Spec $k$ be the scheme-theoretic preimage of $\{0\}$ under the morphism $\phi: U \rightarrow$ $\mathbb{A}_{k}^{n+l}$. Note that $Z=\widetilde{Z}^{\text {red }}$ whence the composition $\widetilde{Z} \rightarrow U \rightarrow \mathbb{A}_{X}^{n}$ is a closed embedding. Since the projection $U \rightarrow \mathbb{A}_{X}^{n}$ is étale then the canonical morphism of $\mathcal{O}_{\widetilde{Z}}$-modules $\rho: J / J^{2} \rightarrow$ $I / I^{2}$ is an isomorphism, where $J$ and $I$ are the sheaves of ideals defining $\widetilde{Z}$ in $U$ and $\mathbb{A}_{X}^{n}$ respectively. The classes $\bar{\phi}_{1}, \ldots, \bar{\phi}_{n+l} \in H^{0}\left(\widetilde{Z}, J / J^{2}\right)$ generate $H^{0}\left(\widetilde{Z}, J / J^{2}\right)$ as an $\mathcal{O}_{\widetilde{Z}}$-module thus $\rho\left(\bar{\phi}_{1}\right), \ldots, \rho\left(\bar{\phi}_{n+l}\right)$ generate $H^{0}\left(\widetilde{Z}, I / I^{2}\right)$ as an $\mathcal{O}_{\widetilde{Z}}$-module. Put

$$
\Theta: \operatorname{Fr}_{n}\left(Y_{+}, X_{+} \wedge T^{l}\right) \rightarrow \operatorname{Fr}_{n}^{\mathcal{V}}\left(Y, X \wedge T^{l}\right)
$$

to be the map given by

$$
\left(Z, U,\left(\phi_{1}, \ldots, \phi_{n+l}\right), g\right) \mapsto\left(\widetilde{Z}, \rho\left(\bar{\phi}_{1}\right), \ldots, \rho\left(\bar{\phi}_{n+l}\right),\left.g\right|_{\widetilde{Z}}\right) .
$$

Note that $\Sigma \circ \Theta=\Theta \circ \Sigma$.

4.3. Definition. Let $X, Y \in \operatorname{Sm}_{k}$ and $p: Y \rightarrow X$ be a finite morphism. Put $l=\operatorname{dim} X-\operatorname{dim} Y$. Denote $\operatorname{Fr}_{n}(X \stackrel{p}{\leftarrow} Y)$ the subset of $\operatorname{Fr}_{n}\left(X_{+}, Y_{+} \wedge T^{l}\right)$ consisting of $(Z, U, \phi, g)$ satisfying:

(1) The diagram

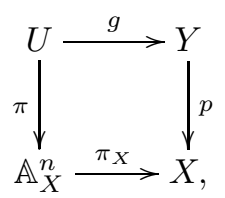

commutes. Here $\pi$ and $\pi_{X}$ are the canonical projections.

(2) For the scheme-theoretic preimage $\widetilde{Z}=\phi^{-1}(0)$ the morphism $g: \widetilde{Z} \rightarrow Y$ is an isomorphism.

We refer to the elements of $\operatorname{Fr}_{n}(X \stackrel{p}{\leftarrow} Y)$ as framed enhancements of $p$.

Denote $\operatorname{Fr}_{n}^{\mathcal{V}}(X \stackrel{p}{\leftarrow} Y)$ the subset $\operatorname{of}_{n}^{\mathcal{V}}\left(X, Y \wedge T^{l}\right)$ consisting of $(Z, \phi, g)$ satisfying:

(1) The diagram

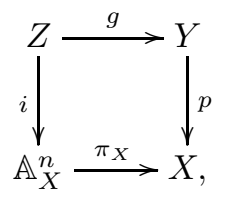

commutes. Here $i$ is the closed embedding and $\pi_{X}$ is the projection.

(2) The morphism $g: Z \rightarrow Y$ is an isomorphism.

In other words, the elements of $\operatorname{Fr}_{n}^{\mathcal{V}}(X \stackrel{p}{\leftarrow} Y)$ are pairs $(i, \phi)$ with $i: Y \rightarrow \mathbb{A}_{X}^{n}$ being a closed embedding over $X$ and $\phi$ being a trivialization of the (co-)normal bundle of $i$.

Note that $\Theta$ from Definition 4.2 can be restricted to a map

$$
\Theta_{p}: \operatorname{Fr}_{n}(X \stackrel{p}{\leftarrow} Y) \rightarrow \operatorname{Fr}_{n}^{\mathcal{V}}(X \stackrel{p}{\leftarrow} Y) .
$$

4.4. Definition. Let $p: Y \rightarrow X$ be a finite map with $X, Y \in \operatorname{Sm}_{k}$. For $a \in \operatorname{Fr}_{n}(X \stackrel{p}{\leftarrow} Y)$ we denote

$$
\operatorname{det} a: \omega_{Y / X} \cong \operatorname{det} N_{i} \stackrel{\simeq}{\rightarrow} \mathcal{O}_{Y}
$$

the trivialization induced by the trivialization $\phi$ with $\Theta_{p}(a)=(i, \phi)$. 
4.5. Lemma. Let $X, Y, V \in \operatorname{Sm}_{k}$ and $p: V \rightarrow Y, q: Y \rightarrow X$ be finite morphisms. Put $l=$ $\operatorname{dim} X-\operatorname{dim} Y$. Then for $a \in \operatorname{Fr}_{n}(X \stackrel{p}{\leftarrow} Y), b \in \operatorname{Fr}_{m}(Y \stackrel{q}{\leftarrow} V)$ one has

$$
\left(b \otimes \operatorname{id}_{T^{l}}\right) \circ a \in \operatorname{Fr}_{n+m}(X \stackrel{q \circ p}{\longleftarrow} V) \text {. }
$$

Proof. Straightforward.

4.6. Lemma. Let $X, Y \in \operatorname{Sm}_{k}$ and $p: Y \rightarrow X$ be a finite morphism. Suppose that $Y$ is affine and that there is an étale map $\pi: Y \rightarrow \mathbb{A}_{k}^{d}$. Then the map

$$
\Theta_{p}: \operatorname{Fr}_{n}(X \stackrel{p}{\leftarrow} Y) \rightarrow \operatorname{Fr}_{n}^{\mathcal{V}}(X \stackrel{p}{\leftarrow} Y)
$$

introduced in Definition 4.2 is surjective.

Proof. Choose $c=(Z, \phi, g) \in \operatorname{Fr}_{n}^{\mathcal{V}}(X \stackrel{p}{\leftarrow} Y)$ and let $I \subset k\left[\mathbb{A}_{X}^{n}\right]$ be the ideal corresponding to $Z$. Take an arbitrary lift of $\phi=\left(\phi_{1}, \ldots, \phi_{n+l}\right) \in H^{0}\left(\mathbb{A}_{X}^{n}, I / I^{2}\right)$ to $\psi=\left(\psi_{1}, \ldots, \psi_{n+l}\right) \in H^{0}\left(\mathbb{A}_{X}^{n}, I\right)$. Note that $I=\left\langle\psi_{1}, \ldots, \psi_{n+l}\right\rangle+I^{2}$ yields that $\left(\psi_{1}, \ldots, \psi_{n+l}\right)$ defines $Z$ in some affine Zariski neighborhood $U^{\prime}$ of $Z$ in $\mathbb{A}_{X}^{n}$.

The composition $\pi \circ g: Z \rightarrow \mathbb{A}_{k}^{d}$ can be extended to a morphism $g^{\prime}: U^{\prime} \rightarrow \mathbb{A}_{k}^{d}$ making the following diagram commute.

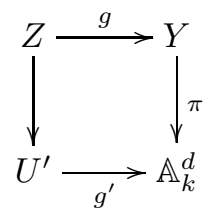

Put $U^{\prime \prime}=U^{\prime} \times_{\mathbb{A}_{k}^{d}} Y$. The morphism $U^{\prime \prime} \rightarrow U^{\prime}$ is étale since $Y \rightarrow \mathbb{A}_{k}^{d}$ is étale. Moreover, $Z \times_{U^{\prime}} U^{\prime \prime}=Z \sqcup Z^{\prime}$ whence $U=U^{\prime \prime}-Z^{\prime}$ is an étale neighborhood of $Z$ in $\mathbb{A}_{X}^{n}$. Then for the projections $\rho_{U^{\prime}}: U \rightarrow U^{\prime}$ and $\rho_{Y}: U \rightarrow Y$ we have

$$
\Theta_{p}\left(Z, U, \psi \circ \rho_{U^{\prime}}, \rho_{Y}\right)=(Z, \phi, g) .
$$

4.7. Lemma. Under the assumptions of Lemma 4.6 suppose that $\Theta_{p}(a)=\Theta_{p}(b)$ for some $a, b \in \operatorname{Fr}_{n}(X \stackrel{p}{\leftarrow} Y)$. Then there exists

$$
H \in \operatorname{Fr}_{n}\left(X \times \mathbb{A}^{1} \stackrel{p \times \operatorname{id}_{\mathbb{A}^{1}}}{\longleftarrow} Y \times \mathbb{A}^{1}\right)
$$

such that $\left.H\right|_{X \times \mathbb{A}^{1}}=a$ and $\left.H\right|_{X \times \mathbb{A}^{1}}=b$.

Proof. Let $a=(Z, U, \phi, g)$ and $b=\left(Z, U^{\prime}, \phi^{\prime}, g^{\prime}\right)$. Passing to the étale neighborhood $U \times_{\mathbb{A}_{X}^{n}} U^{\prime}$ of $Z$ we may assume that $U=U^{\prime}$. Then both the collections of regular functions $\phi, \phi^{\prime}$ generate the same ideal

$$
I=\left\langle\phi_{1}, \ldots, \phi_{n+l}\right\rangle=\left\langle\phi_{1}^{\prime}, \ldots, \phi_{n+l}^{\prime}\right\rangle \text { in } k[U]
$$

and, moreover,

$$
\phi_{i}=\phi_{i}^{\prime} \bmod I^{2}, i=1, \ldots, n+l .
$$

Put $\Phi_{i}=\phi_{i}+t\left(\phi_{i}^{\prime}-\phi_{i}\right) \in k\left[U \times \mathbb{A}^{1}\right]$. Let $J$ denote the ideal $J=I \otimes_{k} k[t]$ in $k\left[U \times \mathbb{A}^{1}\right]$. Then the elements $\Phi_{i}$ lie in $J$ and $\Phi_{i}=\phi_{i} \bmod J^{2}$. Let $J^{\prime}$ denote the ideal $J^{\prime}=\left\langle\Phi_{1}, \ldots, \Phi_{n+l}\right\rangle$. Then $J \cdot J / J^{\prime}=J / J^{\prime}$ and by Nakayama's lemma $r \cdot J / J^{\prime}=0$ for some element $r$ in $k\left[U \times \mathbb{A}^{1}\right]$ such that $r=1 \bmod J$. Then $U \times \mathbb{A}^{1}-Z(r)$ is an open neighborhood of $Z \times \mathbb{A}^{1}$ and we have

$$
Z\left(\Phi_{1}, \ldots, \Phi_{n+l}\right) \cap\left(U \times \mathbb{A}^{1}-Z(r)\right)=\left(Z \times \mathbb{A}^{1}\right),
$$

so

$$
Z(\Phi)=Z\left(\Phi_{1}, \ldots, \Phi_{n+l}\right)=\left(Z \times \mathbb{A}^{1}\right) \sqcup \mathcal{Z}^{\prime}
$$

Put $\mathcal{U}^{\prime}=\left(U \times \mathbb{A}^{1}\right)-\mathcal{Z}^{\prime}$. Note that $\mathcal{Z}^{\prime} \cap(U \times\{0,1\})=\emptyset$, i.e. $U \times\{0,1\} \subset \mathcal{U}^{\prime}$. 
Take an étale map $\pi: Y \rightarrow \mathbb{A}_{k}^{d}$. Similar to the proof of the previous Lemma we can extend

$$
\left(\pi \times \operatorname{id}_{\mathbb{A}^{1}}\right) \circ\left(g \sqcup g^{\prime}\right): U \times\{0,1\} \rightarrow \mathbb{A}_{k}^{d} \times \mathbb{A}^{1}
$$

to a map $G: \mathcal{U}^{\prime} \rightarrow \mathbb{A}_{k}^{d} \times \mathbb{A}^{1}$ making the following diagram commute.

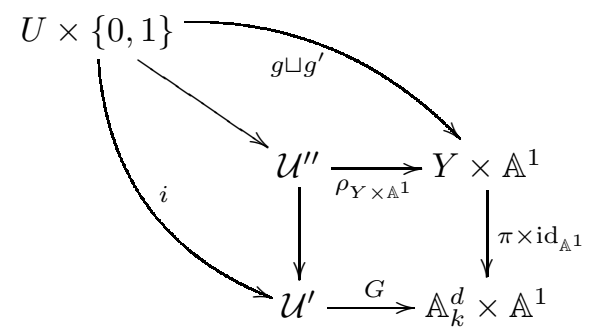

Here $i$ is the closed embedding and $\mathcal{U}^{\prime \prime}=\mathcal{U}^{\prime} \times_{\mathbb{A}_{k}^{d} \times \mathbb{A}^{1}}\left(Y \times \mathbb{A}^{1}\right)$. We have

$$
\left(Z \times \mathbb{A}^{1}\right) \times \mathcal{U}^{\prime} \mathcal{U}^{\prime \prime}=\left(Z \times \mathbb{A}^{1}\right) \sqcup \mathcal{Z}^{\prime \prime} .
$$

Put $\mathcal{U}=\mathcal{U}^{\prime \prime}-\mathcal{Z}^{\prime \prime}$. Then

$$
H=\left(Z \times \mathbb{A}^{1}, \mathcal{U},\left.\Phi\right|_{\mathcal{U}}, \rho_{Y \times \mathbb{A}^{1}} \mid \mathcal{U}\right) \in \operatorname{Fr}_{n}\left(X \times \mathbb{A}^{1} \stackrel{p \times \operatorname{id}_{\mathbb{A}^{1}}}{\longleftarrow} Y \times \mathbb{A}^{1}\right)
$$

gives the claim.

4.8. Definition. For $X, Y \in \operatorname{Sm}_{k}$ and a finite map $p: Y \rightarrow X$ we say that $a, b \in \operatorname{Fr}_{n}(X \stackrel{p}{\leftarrow} Y)$ (resp. $\left.\operatorname{Fr}_{n}^{\mathcal{V}}(X \stackrel{p}{\leftarrow} Y)\right)$ are $\mathbb{A}^{1}$-homotopic if there exists $H \in \operatorname{Fr}_{n}\left(X \times \mathbb{A}^{1} \stackrel{p \times \mathrm{id}_{\mathbb{A}^{1}}}{\longleftarrow} Y \times \mathbb{A}^{1}\right)($ resp. $\left.\operatorname{Fr}_{n}^{\mathcal{V}}\left(X \times \mathbb{A}^{1} \stackrel{p \times \operatorname{id}_{\mathbb{A}^{1}}}{\longleftarrow} Y \times \mathbb{A}^{1}\right)\right)$ such that

$$
\left.H\right|_{X \times\{0\}}=a,\left.\quad H\right|_{X \times\{1\}}=b .
$$

Denote by $\overline{\mathrm{Fr}}_{n}(X \stackrel{p}{\leftarrow} Y)$ (resp. $\left.\overline{\mathrm{Fr}}_{n}^{\mathcal{V}}(X \stackrel{p}{\leftarrow} Y)\right)$ the set of equivalence classes of $\mathbb{A}^{1}$-homotopic correspondences.

4.9. Corollary. Let $X, Y \in \mathrm{Sm}_{k}$ and $p: Y \rightarrow X$ be a finite morphism. Suppose that $Y$ is affine and that there is an étale map $\pi: Y \rightarrow \mathbb{A}_{k}^{d}$, then the map introduced in Definition 4.2 induces a bijection

$$
\Theta_{p}: \overline{\mathrm{Fr}}_{n}(X \stackrel{p}{\leftarrow} Y) \stackrel{\simeq}{\rightarrow} \overline{\mathrm{Fr}}_{n}^{\mathcal{V}}(X \stackrel{p}{\leftarrow} Y) .
$$

Proof. Follows from Lemmas 4.6 and 4.7 .

4.10. Definition. Let $X, Y \in \operatorname{Sm}_{k}$ and $p: Y \rightarrow X$ be a finite morphism. Put $l=\operatorname{dim} X-\operatorname{dim} Y$. For $A \in \mathrm{GL}_{n+l}(k[Y])$ and $(i, \phi) \in \operatorname{Fr}_{n}^{\mathcal{V}}(X \stackrel{p}{\leftarrow} Y)$ put

$$
(i, \phi) \cdot A=(i, \phi \cdot A) \in \operatorname{Fr}_{n}^{\mathcal{V}}(X \stackrel{p}{\leftarrow} Y) .
$$

Here on the right-hand side we take the matrix multiplication of the row $\phi=\left(\phi_{1}, \ldots, \phi_{n+l}\right)$ by the matrix $A$.

4.11. Lemma. Let $X, Y \in \operatorname{Sm}_{k}$ and $p: Y \rightarrow X$ be a finite morphism. Put $l=\operatorname{dim} X-\operatorname{dim} Y$. Suppose that $Y$ is affine. Then for every $(i, \phi),(j, \psi) \in \operatorname{Fr}_{n}^{\mathcal{V}}(X \stackrel{p}{\leftarrow} Y)$ there exists a matrix $A \in \mathrm{GL}_{2 n+l}(k[Y])$ such that

$$
\overline{\Sigma^{n}(i, \phi)}=\overline{\Sigma^{n}(j, \psi) \cdot A} \in \overline{\operatorname{Fr}}_{2 n}^{\mathcal{V}}(X \stackrel{p}{\leftarrow} Y) .
$$


Proof. Consider the map

$$
I: Y \times \mathbb{A}^{1} \rightarrow \mathbb{A}_{X}^{n} \times_{X} \mathbb{A}_{X}^{n} \times \mathbb{A}^{1}=\mathbb{A}_{X}^{2 n} \times \mathbb{A}^{1}
$$

given by the formula

$$
I(y, t)=((1-t) i(y)+t j(y), t(t-1) i(y), t) .
$$

This morphism is a morphism over $X \times \mathbb{A}^{1}$ whence $I$ is finite, moreover, it is clear that $I$ is a monomorphism. Thus $I$ is a closed embedding. Denote by $N_{I}$ the normal bundle for the embedding. Lindel theorem [11] yields that

$$
N_{I} \cong \pi^{*}\left(\left.N_{I}\right|_{Y \times\{0\}}\right)
$$

with $\pi: Y \times \mathbb{A}^{1} \rightarrow Y$ being the projection. Since $\left.I\right|_{Y \times\{0\}}$ is the suspension of inclusion $i$, the pullback of the suspension of $\phi$ gives a trivialization of $N_{I}$ which we denote $\Phi$. We obtained

$$
(I, \Phi) \in \operatorname{Fr}_{2 n}^{\mathcal{V}}\left(X \times \mathbb{A}^{1} \stackrel{p \times \mathrm{id}_{\mathbb{A}^{1}}}{\longleftarrow} \times \mathbb{A}^{1}\right)
$$

with $\left.(I, \Phi)\right|_{X \times\{0\}}=\Sigma^{n}(i, \phi)$ and $\left.(I, \Phi)\right|_{X \times\{1\}}=\left(j \times\{0\}, \psi^{\prime}\right)$ for some trivialization $\psi^{\prime}$ of the normal bundle for the embedding $j \times\{0\}: Y \rightarrow \mathbb{A}_{X}^{n} \times \mathbb{A}^{n}$. The claim follows, since every two trivializations differ by some matrix $A \in \mathrm{GL}_{2 n+l}(k[Y])$.

\section{Pushforward MAPs: FInITE MORPhisms}

5.1. Definition. For $X \in \mathrm{Sm}_{k}$, a closed subset $S \subset X$ and a sheaf of abelian groups $M$ the Cousin complex $C_{S}^{\bullet}(X, M)$ is the complex

$$
\bigoplus_{x \in X^{(0)} \cap S} H_{x}^{0}(X, M) \rightarrow \bigoplus_{x \in X^{(1)} \cap S} H_{x}^{1}(X, M) \rightarrow \ldots \rightarrow \bigoplus_{x \in X^{(d)} \cap S} H_{x}^{d}(X, M)
$$

with the terms given by

$$
C_{S}^{n}(X, M)=\bigoplus_{x \in X^{(n)} \cap S} H_{x}^{n}(X, M)
$$

and the differential induced by the colimit of the connecting homomorphisms

$$
H_{Z-Z^{\prime}}^{n}\left(X-Z^{\prime}, M\right) \rightarrow H_{Z^{\prime}-Z^{\prime \prime}}^{n+1}\left(X-Z^{\prime \prime}, M\right)
$$

for closed subsets $Z^{\prime \prime} \subset Z^{\prime} \subset Z$ [6, §1.2]. For a homotopy module $M_{*}$ [6, Theorem 5.1.10] yields that there are canonical isomorphisms

$$
H_{S}^{n}\left(X, M_{*}\right) \cong H^{n}\left(C_{S}^{\bullet}(X, M)\right) .
$$

5.2. Definition. For this section fix the following notation: $X, Y \in \operatorname{Sm}_{k}, p: Y \rightarrow X$ is a finite morphism, $l=\operatorname{dim} X-\operatorname{dim} Y, M_{*}$ is a homotopy module.

5.3. Definition. In the notation of Definition 5.2 let $Z \subset X$ be a closed subset. Then for $a \in \operatorname{Fr}_{n}(X \stackrel{p}{\leftarrow} Y)$ in the notation of Definition 3.14 we have $a^{-1}\left(p^{-1}(Z)\right)=Z$ and get a homomorphism

$$
a^{*}: H_{p^{-1}(Z)}^{n-l}\left(Y, M_{*-l}\right) \rightarrow H_{Z}^{n}\left(X, M_{*}\right) .
$$

5.4. Lemma. In the notation of Definition 5.2 let $S \subset X$ be a closed subset. Then for every $a \in \operatorname{Fr}_{n}(X \stackrel{p}{\leftarrow} Y)$ the homomorphisms from Definition 5.3 give rise to a morphism of complexes

$$
a^{*}: C_{p^{-1}(S)}^{\bullet}\left(Y, M_{*-l}\right)[-l] \rightarrow C_{S}^{\bullet}\left(X, M_{*}\right) .
$$

This morphism depends only on the class of a in $\overline{\operatorname{Fr}}_{n}(X \stackrel{p}{\leftarrow} Y)$. 
Proof. For closed embeddings $Z^{\prime \prime} \subset Z^{\prime} \subset Z \subset X$ the differentials

$$
\begin{aligned}
H_{p^{-1}\left(Z-Z^{\prime}\right)}^{n}\left(Y-p^{-1}\left(Z^{\prime}\right), M_{*}\right) & \rightarrow H_{p^{-1}\left(Z^{\prime}-Z^{\prime \prime}\right)}^{n+1}\left(Y-p^{-1}\left(Z^{\prime \prime}\right), M_{*}\right), \\
H_{Z-Z^{\prime}}^{n}\left(X-Z^{\prime}, M_{*}\right) & \rightarrow H_{Z^{\prime}-Z^{\prime \prime}}^{n+1}\left(X-Z^{\prime \prime}, M_{*}\right)
\end{aligned}
$$

are induced by the maps

$$
\begin{aligned}
\mathbb{Z}\left[\left(Y-p^{-1}\left(Z^{\prime \prime}\right)\right) /\left(Y-p^{-1}\left(Z^{\prime}\right)\right)\right] & \rightarrow \mathbb{Z}\left[\left(Y-p^{-1}\left(Z^{\prime}\right)\right) /\left(Y-p^{-1}(Z)\right)\right][1], \\
\mathbb{Z}\left[\left(X-Z^{\prime \prime}\right) /\left(X-Z^{\prime}\right)\right] & \rightarrow \mathbb{Z}\left[\left(X-Z^{\prime}\right) /(X-Z)\right][1]
\end{aligned}
$$

respectively. Since $a^{*}$ is induced by a morphism of sheaves (see Definition 5.3) then it fits into the following commutative diagram.

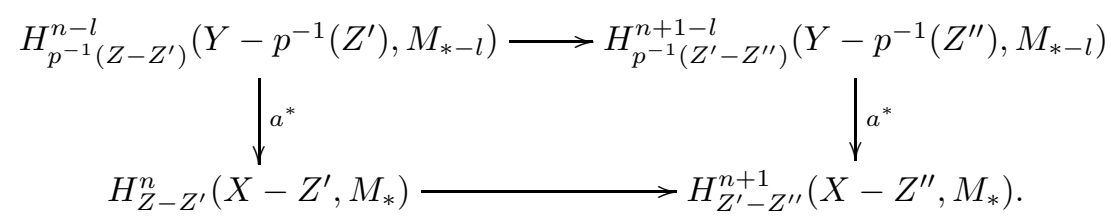

The colimit with respect to $Z^{\prime \prime} \subset Z^{\prime} \subset Z \subset X$ gives the desired morphism of complexes. Since $M_{*}$ is strictly homotopy invariant, $a^{*}$ depends only on the class in $\overline{\operatorname{Fr}}_{n}(X \stackrel{p}{\leftarrow} Y)$.

5.5. Remark. In the notations of the above lemma the assignment

$$
U \mapsto C_{p^{-1}(S)}^{n}\left(p^{-1}(U), M_{*}\right)
$$

defines a sheaf on the small Zariski site of $X$ and $a^{*}$ gives rise to a morphism of complexes

$$
a^{*}: C^{\bullet}\left(p^{-1}(-), M_{*-l}\right)[-l] \rightarrow C_{S}^{\bullet}\left(-, M_{*}\right) .
$$

5.6. Definition. Let $M_{*}$ be a homotopy module. Then Definition 3.15 endows $M_{*}$ with a $\mathrm{GW}$ module structure. For $Y \in \operatorname{Sm}_{k}$ and a line bundle (invertible sheaf) $\mathcal{L}$ on $Y$ define the twist $M_{*} \otimes \mathcal{L}$ as the sheaf on $Y$ given by the tensor product

$$
M_{*} \otimes L=M_{*} \otimes_{\mathbb{Z}\left[\mathcal{O}_{Y}^{\times}\right]} \mathbb{Z}\left[\mathcal{L}^{\times}\right] .
$$

Here

- $\mathcal{O}_{Y}^{\times}$is the sheaf of invertible regular functions,

- $\mathcal{L}^{\times}$is the sheaf of nowhere vanishing sections of $\mathcal{L}$,

- the module structure $M_{*} \times \mathbb{Z}\left[\mathcal{O}_{Y}^{\times}\right] \rightarrow M_{*}$ is induced by the GW-module structure combined with the morphism $\mathcal{O}_{Y}^{\times} \rightarrow \underline{\mathrm{GW}}, u \mapsto\langle u\rangle$.

We are mostly interested in the particular case of

$$
\mathcal{L}=\omega_{Y / X}=\omega_{Y / k} \otimes p^{*} \omega_{X / k}^{-1}
$$

for $X, Y \in \operatorname{Sm}_{k}$ and a morphism $p: Y \rightarrow X$.

5.7. Definition. In the notation of Definition 5.2 for a closed subset $S \subset X$, a line bundle $\mathcal{L}$ on $X$, a trivialization $\theta: \mathcal{L} \stackrel{\simeq}{\rightarrow} \mathcal{O}_{X}$ and $a \in \operatorname{Fr}_{n}(X \stackrel{p}{\leftarrow} Y)$ put

$$
p_{*}^{a, \theta}=\left(\otimes \theta^{-1}\right) \circ a^{*} \circ(\otimes \operatorname{det} a) \circ\left(\otimes p^{*} \theta\right)
$$

for the composition

$$
\begin{aligned}
C_{p^{-1}(S)}^{\bullet}\left(Y, M_{*-l} \otimes \omega_{Y / X} \otimes p^{*} \mathcal{L}\right)[-l] \stackrel{\otimes p^{*} \theta}{\longrightarrow} C_{p^{-1}(S)}^{\bullet}\left(Y, M_{*-l} \otimes \omega_{Y / X}\right)[-l] \stackrel{\otimes \operatorname{det} a}{\longrightarrow} \\
\stackrel{\otimes \operatorname{det} a}{\longrightarrow} C_{p^{-1}(S)}^{\bullet}\left(Y, M_{*-l}\right)[-l] \stackrel{a^{*}}{\longrightarrow} C_{S}^{\bullet}\left(Y, M_{*}\right) \stackrel{\otimes \theta^{-1}}{\longrightarrow} C_{S}^{\bullet}\left(Y, M_{*} \otimes \mathcal{L}\right)
\end{aligned}
$$

Here $\operatorname{det} a: \omega_{Y / X} \stackrel{\simeq}{\rightarrow} \mathcal{O}_{Y}$ is given by Definition 4.4 . 
5.8. Lemma. In the notation of Definition 5.2 let $\mathcal{L}$ be a trivial line bundle on $X$. Suppose that $Y$ is affine an that there exists an étale map $Y \rightarrow \mathbb{A}_{k}^{d}$. Then for $a, b \in \operatorname{Fr}_{n}(X \stackrel{p}{\leftarrow} Y)$ and $\theta, \theta^{\prime}: \mathcal{L} \stackrel{\simeq}{\rightarrow} \mathcal{O}_{X}$ we have

$$
p_{*}^{a, \theta}=p_{*}^{b, \theta^{\prime}}: C_{p^{-1}(S)}^{\bullet}\left(Y, M_{*-l} \otimes \omega_{Y / X}\right)[-l] \rightarrow C_{S}^{*}\left(X, M_{*}\right) .
$$

Proof. The equality $p_{*}^{a, \theta}=p_{*}^{a, \theta^{\prime}}$ follows from Lemma 3.16 claiming $a^{*}$ to be a homomorphism of $\underline{\mathrm{GW}}$-modules. The equality $p_{*}^{a, \theta^{\prime}}=p_{*}^{b, \theta^{\prime}}$ follows from the fact that $a^{*}$ depends only on the $\mathbb{A}^{1}$-homotopy class of $a$ and Corollary 4.9 combined with Lemmas 3.18 and 4.11.

5.9. Lemma. For $X \in \mathrm{Sm}_{k}$ and a finite collection of points $x_{1}, \ldots, x_{n} \in X$ there exists a Zariski open $U \subset X, x_{i} \in U, i=1, \ldots n$, and an étale map $f: U \rightarrow \mathbb{A}_{k}^{d}$ with $d=\operatorname{dim} X$.

Proof. A general projection $U \rightarrow \mathbb{A}_{k}^{d}$ of an affine Zariski open $U \subset X, x_{i} \in U, i=1, \ldots n$, is étale at $x_{1}, \ldots, x_{n}$ whence the claim.

5.10. Definition. In the notation of Definition 5.2 let $S \subset X$ be a closed subset and $\mathcal{L}$ be a line bundle on $X$. Define the pushforward map

$$
p_{*}: C_{p^{-1}(S)}^{\bullet}\left(Y, M_{*-l} \otimes \omega_{Y / X} \otimes p^{*} \mathcal{L}\right)[-l] \rightarrow C_{S}^{\bullet}\left(X, M_{*} \otimes \mathcal{L}\right)
$$

as follows. By Lemma 5.9 we can find an open cover $\bigcup_{i=1}^{n} U_{i}=X$ such that for every $i$ the following holds:

(1) $U_{i}$ is affine,

(2) $\left.\mathcal{L}\right|_{U_{i}}$ is trivial,

(3) $V_{i}=p^{-1}\left(U_{i}\right)$ admits an étale map $V_{i} \rightarrow \mathbb{A}_{k}^{d}$,

(4) there exists a closed embedding $V_{i} \rightarrow \mathbb{A}_{U_{i}}^{n}$ over $U_{i}$ with a trivial normal bundle.

These conditions imply that we can choose some $a_{i} \in \operatorname{Fr}_{n}\left(U_{i} \stackrel{p_{i}}{\longleftarrow} V_{i}\right)$ together with trivializations $\theta_{i}:\left.\mathcal{L}\right|_{U_{i}} \stackrel{\simeq}{\rightarrow} \mathcal{O}_{U_{i}}$ with $p_{i}=\left.p\right|_{V_{i}}$. Lemma 5.8 yields that the maps

$$
p_{i *}=\left(p_{i}^{a_{i}, \theta_{i}}\right)_{*}: C^{\bullet}\left(V_{i}, M_{*-l} \otimes \omega_{V_{i} / U_{i}} \otimes p^{*}(\mathcal{L})\right)[-l] \rightarrow C^{\bullet}\left(U_{i}, M_{*} \otimes \mathcal{L}\right)
$$

do not depend on the choice of $a_{i}$ and $\theta_{i}$ and, in particular, coincide on the intersections $V_{i j}=$ $V_{i} \cap V_{j}$. Then $p_{i *}$-s give rise to the pushforward map $p_{*}$ making the following diagram commute.

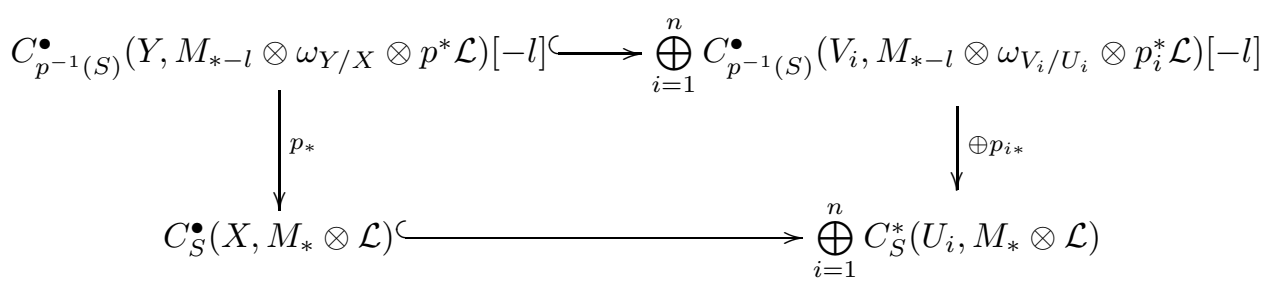

It also follows from Lemma 5.8 that $p_{*}$ does not depend on the cover.

5.11. Lemma. In the notation of Definition 5.2 let $V \in \operatorname{Sm}_{k}$ and $q: V \rightarrow Y$ be a finite morphism. Put $l^{\prime}=\operatorname{dim} X-\operatorname{dim} Y$. Then for a line bundle $\mathcal{L}$ over $X$ and a closed subset $S \subset X$ one has

$$
(p \circ q)_{*}=p_{*} \circ q_{*}: C_{(p \circ q)^{-1}(S)}^{\bullet}\left(V, M_{*-l-l^{\prime}} \otimes \omega_{V / X} \otimes(p \circ q)^{*} \mathcal{L}\right)\left[-l-l^{\prime}\right] \rightarrow C_{S}^{\bullet}\left(X, M_{*} \otimes \mathcal{L}\right) .
$$

Proof. Follows from Lemma 4.5 and the construction.

5.12. Lemma. In the notation of Definition 5.2 for a closed subset $S \subset Y$ the pushforward map

$$
i_{*}: C_{S}^{\bullet}\left(Y, M_{*-l} \otimes \omega_{Y / X}\right)[-l] \rightarrow C_{S}^{\bullet}\left(X, M_{*}\right)
$$

is an isomorphism. 
Proof. Since $i_{*}$ is a morphism of complexes of Zariski sheaves we may shrink $X$. Thus we may assume that there exists $a \in \operatorname{Fr}_{0}(X \stackrel{i}{\leftarrow} Y)$ that induces an isomorphism of sheaves $X /(X-$ $Y) \stackrel{\simeq}{\rightarrow} Y_{+} \wedge T^{l}$. Then for every closed $Z \subset Y$ the map $a^{*}: H_{Z}^{i-l}\left(Y, M_{*-l}\right) \rightarrow H_{Z}^{i}\left(X, M_{*}\right)$ is an isomorphism. Taking the colimit along $Z$ we obtain the claim.

5.13. Corollary. For $X \in \mathrm{Sm}_{k}, x \in X^{(n)}$, a line bundle $\mathcal{L}$ on $X$ and a homotopy module $M_{*}$ the pushforward map

$$
\left(i_{x}\right)_{*}: H^{0}\left(x, M_{*-n} \otimes \omega_{x / X} \otimes i_{x}^{*} \mathcal{L}\right) \stackrel{\simeq}{\rightarrow} H_{x}^{n}\left(X, M_{*} \otimes \mathcal{L}\right)
$$

for the embedding $i_{x}: x \rightarrow X$ is an isomorphism.

Proof. There is a Zariski open $x \in U \subset X$ such that $S=\bar{x} \cap U$ is smooth. Lemma 5.12 applied to the closed embedding $i: S \rightarrow U$ yields an isomorphism

$$
i_{*}: C_{S}^{\bullet}\left(S, M_{*-n} \otimes \omega_{S / U} \otimes i^{*} \mathcal{L}\right)[-n] \rightarrow C_{S}^{\bullet}\left(U, M_{*}\right)
$$

which restricts to the desired isomorphism.

5.14. Corollary. For $X \in \mathrm{Sm}_{k}$, a closed subset $S \subset X$, a line bundle $\mathcal{L}$ on $X$ and a homotopy module $M_{*}$ the pushforward map

$$
\left(i_{\mathcal{L}}\right)_{*}: H_{S}^{n}\left(X, M_{*} \otimes \mathcal{L}\right) \rightarrow H_{S}^{n+1}\left(L, M_{*+1}\right)
$$

is an isomorphism. Here $L$ is the total space of $\mathcal{L}$ and $i_{\mathcal{L}}: X \rightarrow L$ is the zero section. Moreover, if $\theta: \mathcal{L} \stackrel{\simeq}{\rightarrow} \mathcal{O}_{X}$ is a trivialization of $\mathcal{L}$ then the following diagram commutes.

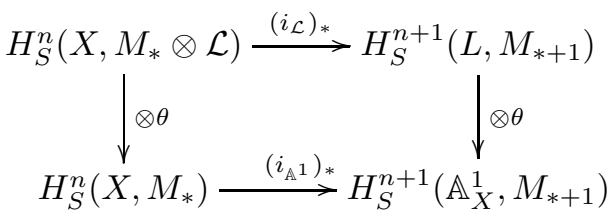

Proof. Follows from Lemma 5.12. The commutativity of the diagram immediately follows from the definition of the pushforward map.

\section{Pushforward maps: General CASe}

6.1. Definition. Let $f: Y \rightarrow X$ be a morphism in $\operatorname{Sm}_{k}$ of relative dimension $d=\operatorname{dim} Y-\operatorname{dim} X$ and let $\mathcal{L}$ be a line bundle on $X$. For $y \in Y^{(n)}$ such that $f: y \rightarrow f(y)$ is finite define $\left(f_{*}\right)_{y}$ to be the morphism making the following diagram commute.

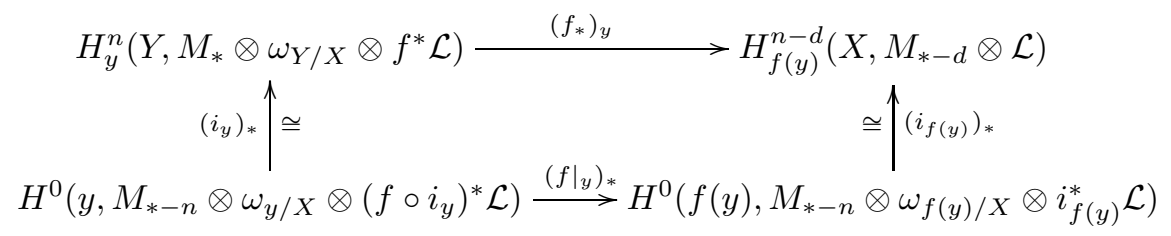

Here the vertical isomorphisms are given by Corollary 5.13 and the bottom morphism is the pushforward morphism from Definition 5.10 for the finite morphism $\left.f\right|_{y}: y \rightarrow f(y)$.

6.2. Proposition. In the notation of Definition 6.1 let $S$ be a closed subset of $Y$ finite over $X$. Then the maps $\left(f_{*}\right)_{y}$ for $y \in S$ define a morphism of complexes

$$
f_{*}: C_{S}^{\bullet}\left(Y, M_{*} \otimes \omega_{Y / X} \otimes f^{*} \mathcal{L}\right) \rightarrow C_{f(S)}^{\bullet}\left(X, M_{*-d} \otimes \mathcal{L}\right)[-d] .
$$


Proof. Consider the case of trivial $\mathcal{L}$, the proof in the general case is the same.

Take $x \in X^{(n-d)} \cap f(S), x^{\prime} \in X^{(n-d+1)} \cap \bar{x}$, choose a point $y \in f^{-1}(x) \cap S$ and let $y_{1}^{\prime}, y_{2}^{\prime}, \ldots, y_{m}^{\prime}$ be the preimages of $x^{\prime}$ in $\bar{y}$. We need to check that the diagram

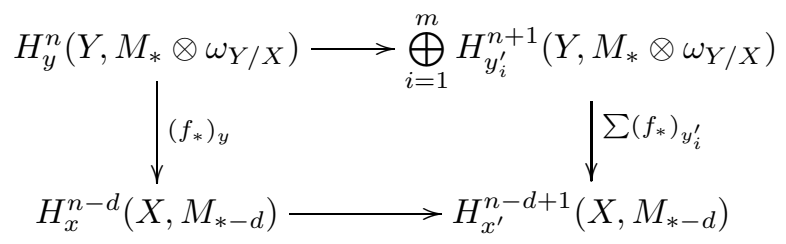

commutes. Let $Z \rightarrow \bar{y}$ be the normalization. There exists Zariski open subsets $U \subset Y$ and $V \subset X$ such that
(1) $y_{i}^{\prime} \in U$ for all $i$,
(3) $\widetilde{Z}=Z \times_{Y} U$ is smooth,
(2) $x^{\prime} \in V$

(4) $f(\bar{y} \cap U)=\bar{x} \cap V$.

Then $p: \widetilde{Z} \rightarrow U$ and $f \circ p: \widetilde{Z} \rightarrow V$ are finite maps between smooth varieties and Definition 5.10 gives morphisms of complexes

$$
\begin{gathered}
p_{*}: C^{\bullet}\left(\widetilde{Z}, M_{*-n} \otimes \omega_{\widetilde{Z} / X}\right)[-n] \rightarrow C^{\bullet}\left(U, M_{*} \otimes \omega_{U / X}\right), \\
(f \circ p)_{*}: C^{\bullet}\left(\widetilde{Z}, M_{*-n} \otimes \omega_{\widetilde{Z} / X}\right)[-n] \rightarrow C^{\bullet}\left(V, M_{*-d}\right)[-d] .
\end{gathered}
$$

Then the squares in the diagram

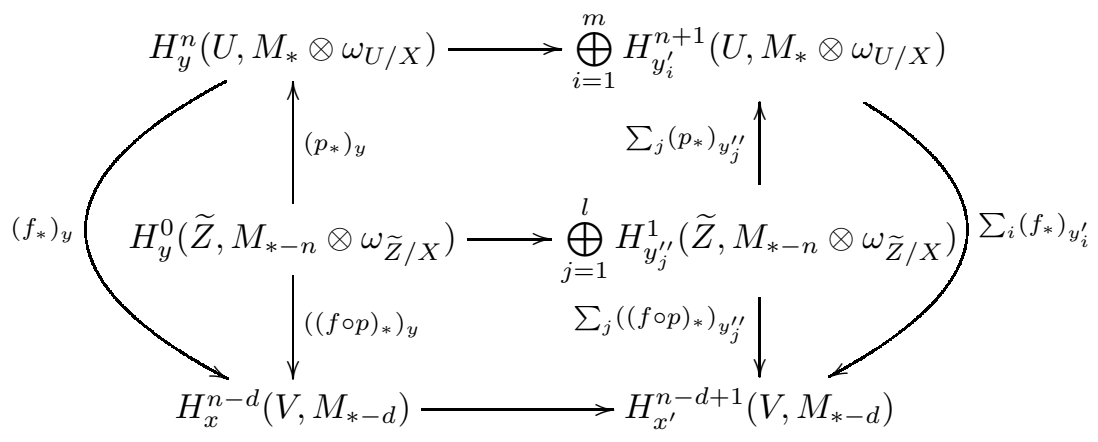

commute. Here $y_{j}^{\prime \prime}$ denote the points of $\widetilde{Z}$ lying over $y_{i}^{\prime}$. The triangles commute by Lemma 5.11 . The claim follows since

$$
\left(p_{*}\right)_{y}: H_{y}^{0}\left(\widetilde{Z}, M_{*-n} \otimes \omega_{\widetilde{Z} / X}\right) \rightarrow H_{y}^{n}\left(U, M_{*} \otimes \omega_{U / X}\right)
$$

is an isomorphism.

6.3. Definition. In the notation of Definition 6.1 let $S$ be a closed subset of $Y$ finite over $X$. Then applying Proposition 6.2 and taking cohomology we obtain pushforward maps

$$
f_{*}: H_{S}^{n}\left(Y, M_{*} \otimes \omega_{Y / X} \otimes f^{*} \mathcal{L}\right) \rightarrow H_{f(S)}^{n-d}\left(X, M_{*-d} \otimes \mathcal{L}\right) .
$$

The constructed pushforward maps are functorial by Lemma 5.11 . 
6.4. Lemma. In the notation of Definition 6.1 let $S$ be a closed subset of $Y$ finite over $X$. Then for an open embedding $j_{X}: X_{0} \rightarrow X$ the square

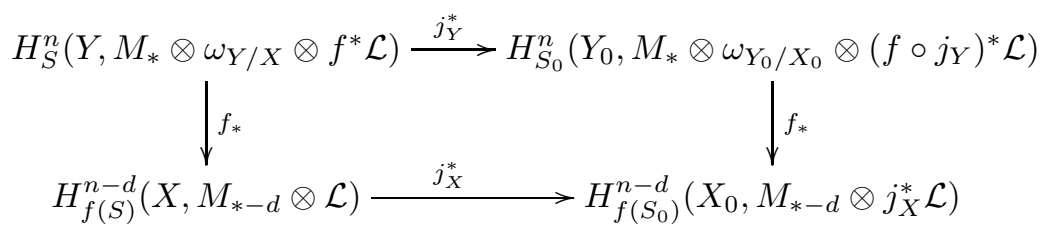

commutes, where $j_{Y}: Y_{0}=X_{0} \times_{X} Y \rightarrow Y$ is the open embedding and $S_{0}=X_{0} \times_{X} S$.

Proof. The claim follows from the commutativity of the following diagram consisting of Cousin complexes which is immediate.

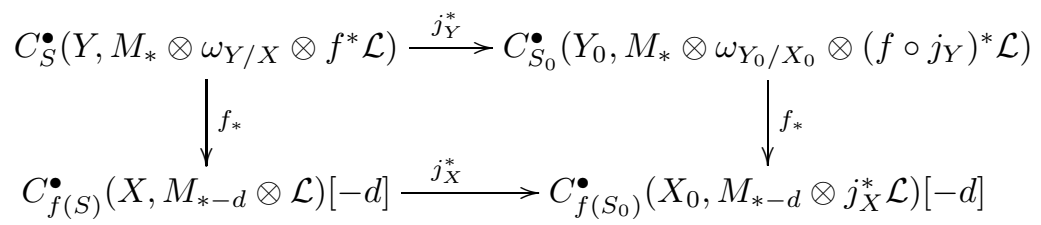

Here $j_{X}^{*}$ is identity on $H_{x}^{i}\left(X, M_{*-d} \otimes \mathcal{L}\right)$ if $x \in X_{0}$ and zero otherwise, and similarly for $j_{Y}^{*}$.

6.5. Remark. It seems that the pushforward maps constructed in Definition 6.1 coincide with the ones introduced in [13, Chapter 5]. We do not need this fact in this paper so we do not check the details.

6.6. Definition. Let $X, Y \in \operatorname{Sm}_{k}$. We say that

(1) a closed embedding $i: Y \rightarrow X$ admits a framed enhancement if $\operatorname{Fr}_{0}(X \stackrel{i}{\leftarrow} Y) \neq \emptyset$,

(2) a morphism $f: Y \rightarrow X$ has a framed enhancement if for some $m$ there exists a Zariski open subset $U \subset \mathbb{A}_{X}^{m}$ and a closed embedding $i: Y \rightarrow U$ over $X$ such that $i$ admits a framed enhancement.

6.7. Remark. If $f: Y \rightarrow X$ admits a framed enhancement then

(1) for every $V \in \operatorname{Sm}_{k}$ the morphism $f \times \operatorname{id}_{V}: Y \times V \rightarrow X \times V$ admits a framed enhancement,

(2) for any Zariski open subset $Y_{0} \subset Y$ the morphism $\left.f\right|_{Y_{0}}: Y_{0} \rightarrow X$ admits a framed enhancement.

6.8. Lemma. For $X \in \operatorname{Sm}_{k}$ and any finite collection of points $x_{1}, \ldots, x_{n} \in X$ there exists a Zariski open $U \subset X$ such $x_{i} \in U$ for all $i$ and the morphism $U \rightarrow$ Spec $k$ admits a framed enhancement.

Proof. Applying Lemma 5.9 choose a Zariski open $U \subset X$ such $x_{i} \in U$ for all $i$ and that there exists an étale map $g: U \rightarrow \mathbb{A}_{k}^{d}$. Choose a closed embedding $i: U \rightarrow \mathbb{A}_{k}^{m}$. The normal bundle $N_{i} \cong\left(\left.T_{\mathbb{A}_{k}^{m}}\right|_{U}\right) / T_{U}$ is stably trivial. Composing $i$ with the closed embedding $\mathbb{A}_{k}^{m} \rightarrow \mathbb{A}_{k}^{m+n}$, $t \mapsto(t, 0)$, for $n$ big enough we may assume that the tangent bundle $N_{i}$ is trivial and $U$ is closed in $\mathbb{A}_{k}^{m}$. Thus $\operatorname{Fr}_{0}^{\mathcal{V}}\left(\mathbb{A}_{k}^{m} \stackrel{i}{\leftarrow} U\right) \neq \emptyset$. Applying Lemma 4.6 we get the claim.

6.9. Definition. Let $U \subset \mathbb{A}_{X}^{m}, a \in \operatorname{Fr}_{0}(U \stackrel{i}{\leftarrow} Y)$ be a framed enhancement of a relative dimension $d=\operatorname{dim} Y-\operatorname{dim} X$ morphism $f: Y \rightarrow X$. Then for every closed subset $S \subset Y$ finite over $X$ the morphism

$$
a: U /(U-i(Y)) \rightarrow Y_{+} \wedge T^{m-d}
$$

gives rise to a morphism of sheaves

$a_{f}: X /(X-f(S)) \wedge \mathbb{P}^{\wedge m} \rightarrow X_{+} \wedge \mathbb{P}^{\wedge m} /\left(X_{+} \wedge \mathbb{P}^{\wedge m}-i(S)\right) \stackrel{\simeq}{\longleftarrow} U /(U-i(S)) \rightarrow Y /(Y-S) \wedge T^{m-d}$. 
6.10. Lemma. Let $M_{*}$ be a homotopy module. Then in the notation of Definition 6.9 the following diagram commutes.

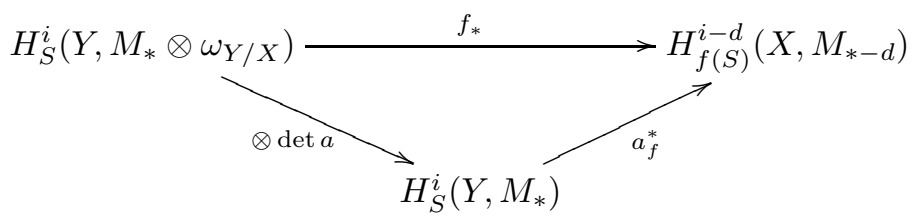

Here $\operatorname{det} a$ is given by Definition 4.4 and $a_{f}^{*}$ is induced by the pullback along $a_{f}$ similar to Definition 3.14.

Proof. For every closed subset $Z \subset S$ the morphism $a_{f}$ induces a morphism

$$
a_{f}^{Z}: X /(X-f(Z)) \wedge \mathbb{P}^{\wedge m} \rightarrow Y /(Y-Z) \wedge T^{m-d}
$$

giving a homomorphism

$$
\left(a_{f}^{Z}\right)^{*}: H_{Z}^{i}\left(Y, M_{*}\right) \rightarrow H_{f(Z)}^{i-d}\left(X, M_{*-d}\right) .
$$

Taking the respective colimit we obtain a morphism of Cousin complexes

$$
a_{f}^{*}: C_{S}^{\bullet}\left(Y, M_{*}\right) \rightarrow C_{f(S)}^{\bullet}\left(X, M_{*-d}\right)[-d]
$$

inducing the map $a_{f}^{*}$ from the statement of the lemma.

It is sufficient to check that for every $y \in Y^{(n)} \cap S$ the following diagram commutes.

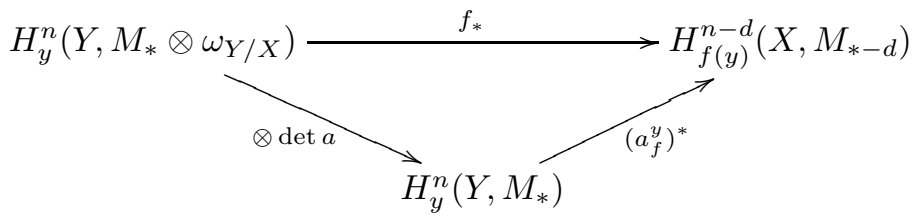

For a point $y \in Y^{(n)} \cap S$ choose a Zariski open subset $y \in U \subset Y$ such that $V=\bar{y} \cap U$ is smooth and the closed embedding $i: V \rightarrow U$ admits a framed enhancement $c \in \operatorname{Fr}_{0}(U \stackrel{i}{\leftarrow} V)$. For $W=f(U)$ the correspondence $a_{f}$ induces morphism

$$
a_{f}^{V}: W /(W-f(V)) \wedge \mathbb{P}^{\wedge m} \rightarrow U /(U-V) \wedge T^{m-d} .
$$

Then $c \circ a_{f}^{V} \in \operatorname{Fr}_{m}(W \stackrel{f \circ i}{\longleftarrow} V)$ is a framed enhancement of the finite map $f \circ i: V \rightarrow W$. Consider the following diagram.

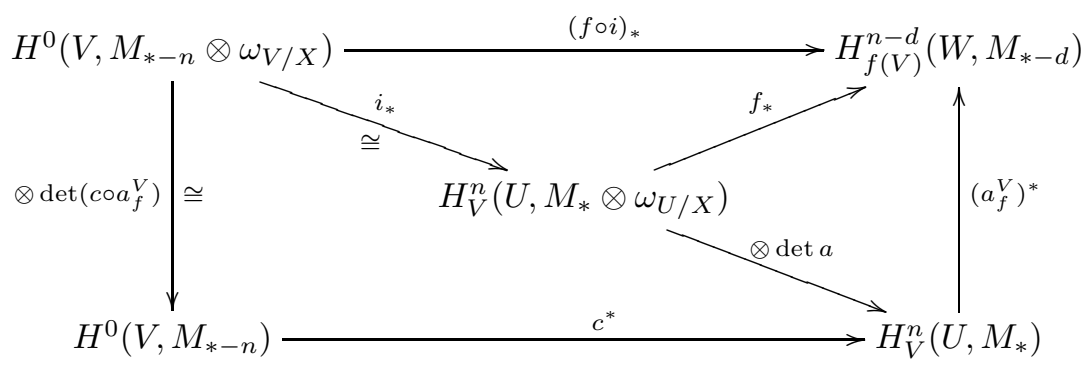

The outer contour and the lower square commute by the definition of pushforward maps for finite morphism. The upper triangle commutes by functoriality of pushforward maps. The map $i_{*}$ is an isomorphism by Lemma 5.12. Thus the right triangle commutes as well and taking colimits along neighborhoods of $y$ we obtain the claim. 
6.11. Lemma. Let $f: Y \rightarrow X$ be a morphism in $\operatorname{Sm}_{k}$ of relative dimension $d=\operatorname{dim} Y-\operatorname{dim} X$. Suppose that $f$ admits a framed enhancement. Then for $c \in \operatorname{Fr}_{n}(V, W)$ and a closed subset $S \subset W \times Y$ finite over $W \times X$ the following diagram commutes.

$$
\begin{gathered}
H_{S}^{i}\left(W \times Y, M_{*} \otimes \omega_{Y / X}\right) \stackrel{\left(c \unrhd \operatorname{id}_{X}\right)^{*}}{\longrightarrow} H_{c^{-1}(S)}^{i}\left(V \times Y, M_{*} \otimes \omega_{Y / X}\right) \\
\downarrow \begin{array}{l}
\left(\operatorname{id}_{W} \times f\right)_{*} \\
\left(\operatorname{id}_{V} \times f\right)_{*}
\end{array} \\
H_{f(S)}^{i-d}\left(W \times X, M_{*-d}\right) \stackrel{\left(c \otimes \operatorname{id}_{Y}\right)^{*}}{\longrightarrow} H_{\left(f \times c^{-1}\right)(S)}^{i-d}\left(V \times X, M_{*-d}\right)
\end{gathered}
$$

Here were shortened the notation as

$c^{-1}(S)=\left(c \otimes \operatorname{id}_{X}\right)^{-1}(S), \quad f(S)=\left(\operatorname{id}_{W} \times f\right)(S), \quad\left(f \times c^{-1}\right)(S)=\left(\operatorname{id}_{V} \times f\right)\left(\left(c \otimes \operatorname{id}_{X}\right)^{-1}(S)\right)$.

Proof. Let $U \subset \mathbb{A}_{X}^{m}, a \in \operatorname{Fr}_{0}(U \stackrel{i}{\leftarrow} Y)$ be a framed enhancement of $f$. Lemma 6.10 yields that

$$
\left(\operatorname{id}_{W} \times f\right)_{*}=\left(a_{\operatorname{id}_{W} \times f}\right)^{*} \circ(\otimes \operatorname{det} a), \quad\left(\operatorname{id}_{V} \times f\right)_{*}=\left(a_{\operatorname{id}_{V} \times f}\right)^{*} \circ(\otimes \operatorname{det} a) .
$$

The claim follows from the commutativity of the following diagram which is straightforward.

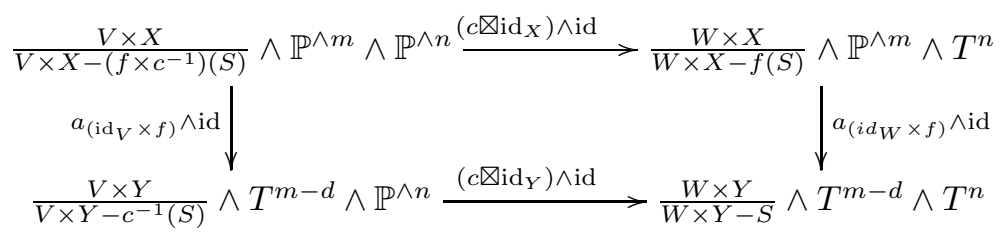

6.12. Lemma. Let $f: Y \rightarrow X$ be a morphism in $\operatorname{Sm}_{k}$ of relative dimension $d=\operatorname{dim} Y-\operatorname{dim} X$ and $S \subset Y$ be a closed subset finite over $X$. Suppose that $f$ admits a framed enhancement. Then for a homotopy module $M_{*}$ and $c \in H_{S^{\prime}}^{j}\left(V, \mathrm{~K}_{n}^{\mathrm{MW}}\right)$ the following diagram commutes.

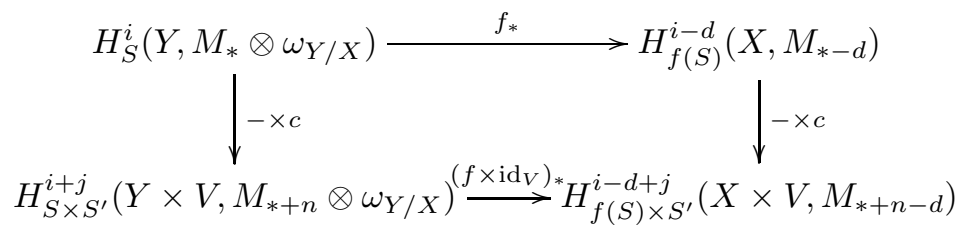

Proof. Let $U \subset \mathbb{A}_{X}^{m}, a \in \operatorname{Fr}_{0}(U \stackrel{i}{\leftarrow} Y)$ be a framed enhancement of $f$. Lemma 6.10 yields that

$$
f_{*}=a_{f}^{*} \circ(\otimes \operatorname{det} a), \quad\left(f \times \operatorname{id}_{V}\right)_{*}=a_{f \times \operatorname{id}_{V}}^{*} \circ(\otimes \operatorname{det} a) .
$$

Moreover, $a_{f \times \mathrm{id}_{V}}=a_{f} \otimes \mathrm{id}_{V / V-S^{\prime}}$.

For $m \in H_{S}^{i}\left(Y, M_{*}\right)$ given by a morphism

$$
m: \mathbb{Z}[Y / Y-S] \rightarrow M_{*}[i]
$$

in $\mathrm{D}(\operatorname{Shv} \mathrm{Ab}(k))$ the external product $m \times c$ is given by the map

$$
m \times c: \mathbb{Z}[Y / Y-S] \otimes \mathbb{Z}\left[V / V-S^{\prime}\right] \rightarrow M_{*}[i] \times \mathrm{K}_{n}^{\mathrm{MW}}[j] \rightarrow M_{*+n}[i+j] .
$$

Then

$$
\left(f \times \operatorname{id}_{V}\right)_{*}(m \times c)=a_{f \times \operatorname{id}_{V}} \circ(m \times c)=\left(a_{f} \circ m\right) \times c=f_{*}(m) \times c
$$

and the claim follows. 


\section{Milnor-Witt CORRESPONDENCES}

7.1. Definition. [4, §4] For $X, Y \in \operatorname{Sm}_{k}$ with $\operatorname{dim} Y=d$ and a closed subset $S \subset X \times Y$ put

$$
\widetilde{\operatorname{Cor}}_{S}(X, Y)=H_{S}^{d}\left(X \times Y, \mathrm{~K}_{d}^{\mathrm{MW}} \otimes \omega_{Y}\right)
$$

The group of finite MW-correspondences is defined as

$$
\widetilde{\operatorname{Cor}}(X, Y)=\operatorname{colim}_{S} \widetilde{\operatorname{Cor}_{S}}(X, Y)
$$

where the colimit is taken along all the admissible closed subsets $S \subset X \times Y$, i.e. the closed subsets $S \subset X \times Y$ such that every irreducible component of $S$ is finite and surjective over some irreducible component of $X$. See $[4, \S 4.2]$ for the definition of composition of finite MWcorrespondences leading to the category $\widetilde{\mathrm{Cor}}_{k}$ with the objects those of $\mathrm{Sm}_{k}$ and the morphisms given by $\widetilde{\operatorname{Cor}}(X, Y)$. An additive contravariant functor $\widetilde{\operatorname{Cor}}_{k} \rightarrow \mathrm{Ab}$ is called a presheaf with MW-transfers.

7.2. Remark. For $X, Y \in \operatorname{Sm}_{k}$ with $\operatorname{dim} Y=d$ and a closed subset $S \subset X \times Y$ let $L_{Y}$ be the total space of the canonical line bundle $\omega_{Y}$ and $i: X \times Y \rightarrow X \times L_{Y}$ be the zero section. Then

$$
i_{*}: \widetilde{\operatorname{Cor}}_{S}(X, Y)=H_{S}^{d}\left(X \times Y, \mathrm{~K}_{d}^{\mathrm{MW}} \otimes \omega_{Y}\right) \stackrel{\simeq}{\rightarrow} H_{S}^{d+1}\left(X \times L_{Y}, \mathrm{~K}_{d+1}^{\mathrm{MW}}\right) .
$$

is an isomorphism by Corollary 5.14 .

7.3. Definition. Let $X, Y, V \in \operatorname{Sm}_{k}$ and $S \subset X \times Y$ be a closed subset. Then every $a \in \operatorname{Fr}_{n}(V, X)$ defines a unique homomorphism $a^{*}$ making the following diagram commute.

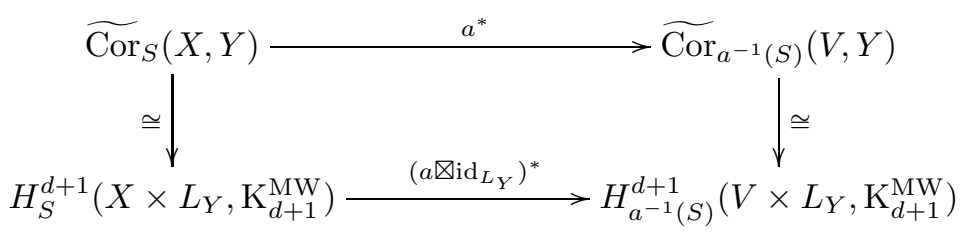

Here $a^{-1}(S)=\left(a \otimes \operatorname{id}_{Y}\right)^{-1}(S)$, the vertical isomorphisms are given by Remark 7.2 and the bottom morphism is the pullback on the cohomology introduced in Definition 3.14.

Note that if $S \subset X \times Y$ is admissible then $a^{-1}(S) \subset V \times Y$ is admissible as well. The homomorphism $a^{*}$ commutes with the inclusions of admissible subsets and gives rise to a homomorphism

$$
a^{*}: \widetilde{\operatorname{Cor}}(X, Y) \rightarrow \widetilde{\operatorname{Cor}}(V, Y) .
$$

It is straightforward to check that $(a \circ b)^{*}=b^{*} \circ a^{*}$ for $W \in \operatorname{Sm}_{k}$ and $b \in \operatorname{Fr}_{m}(W, V)$. Then the above rule endows $\widetilde{\operatorname{Cor}}(-, Y)$ with the structure of a framed presheaf. Lemma 2.10 yields that this structure descends to the structure of a $\mathbb{Z F} F_{*}$-presheaf and gives rise to a homomorphism

$$
\mathcal{D}: \mathbb{Z F}_{*}(X, Y) \rightarrow \widetilde{\operatorname{Cor}}(X, Y), \quad \mathcal{D}(a)=a^{*}\left(1_{Y}\right)
$$

where $1_{Y} \in \widetilde{\operatorname{Cor}}(Y, Y)$ is the identity morphism. 
7.4. Lemma. In the notation of Definition 7.3 suppose that $\omega_{Y}$ is trivial and fix a trivialization $\theta: \omega_{Y} \stackrel{\simeq}{\rightarrow} \mathcal{O}_{Y}$. Then the following diagram commutes.

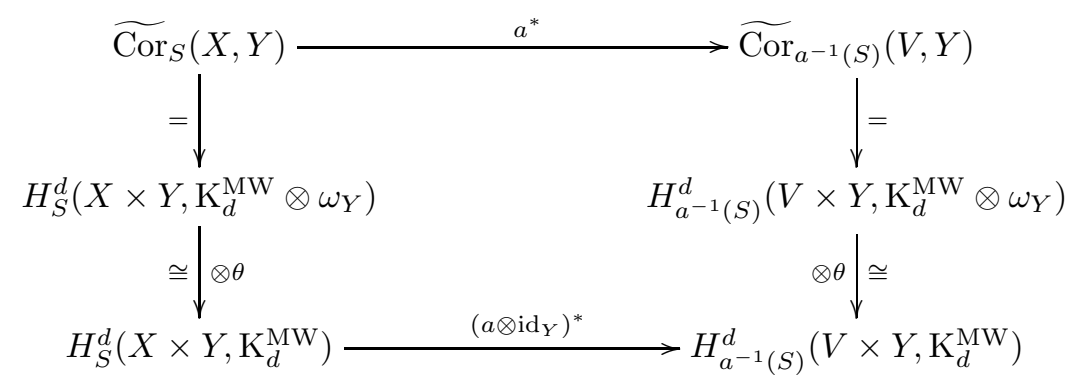

Proof. Follows from Corollary 5.14.

7.5. Definition. Let $X, Y, V \in \mathrm{Sm}_{k}, S \subset V \times X$ be an admissible closed subset and $Z \subset X \times Y$ be a closed subset. Denote $p: V \times X \times Y \rightarrow V \times Y$ the projection and put $d=\operatorname{dim} X$. For a homotopy module $M_{*}$ define a pairing

$$
\widetilde{\operatorname{Cor}}_{S}(V, X) \times H_{Z}^{i}\left(X \times Y, M_{*}\right) \stackrel{\cup}{\rightarrow} H_{S \cdot Z}^{i}\left(V \times Y, M_{*}\right)
$$

with $S \cdot Z=p(S \times Y \cap V \times Z)$ as the following composition

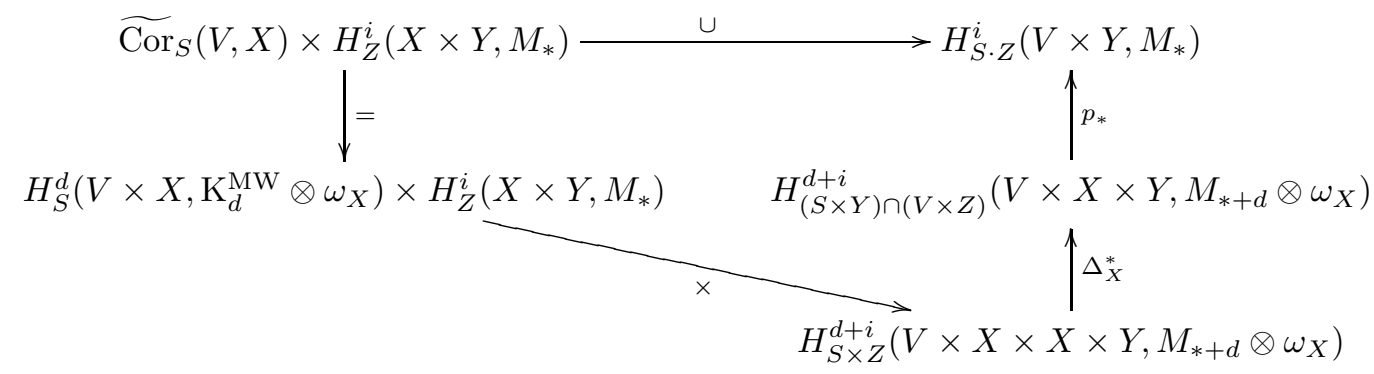

7.6. Lemma. In the notation of Definition 7.5 suppose that $X \rightarrow$ Spec $k$ admits a framed enhancement. Then for every $W \in \operatorname{Sm}_{k}$ and $a \in \operatorname{Fr}_{n}(W, V)$ the following square commutes.

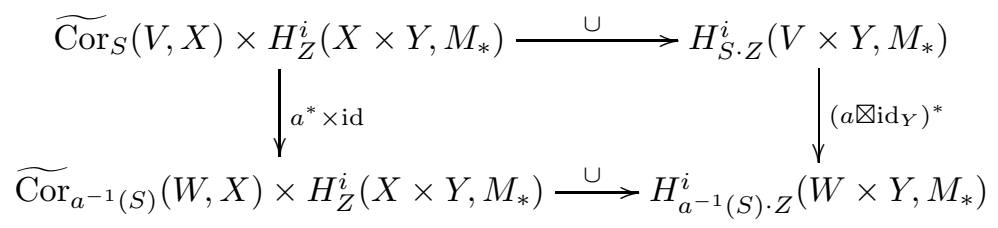

Proof. Recall that

$$
\widetilde{\operatorname{Cor}_{S}}(V, X)=H_{S}^{d}\left(V \times X, \mathrm{~K}_{d}^{\mathrm{MW}} \otimes \omega_{X}\right), \quad \widetilde{\operatorname{Cor}_{a^{-1}(S)}}(W, X)=H_{a^{-1}(S)}^{d}\left(W \times X, \mathrm{~K}_{d}^{\mathrm{MW}} \otimes \omega_{X}\right) .
$$


Then for every $b \in H_{Z}^{i}\left(X \times Y, M_{*}\right)$ we need to prove commutativity of the following diagram.

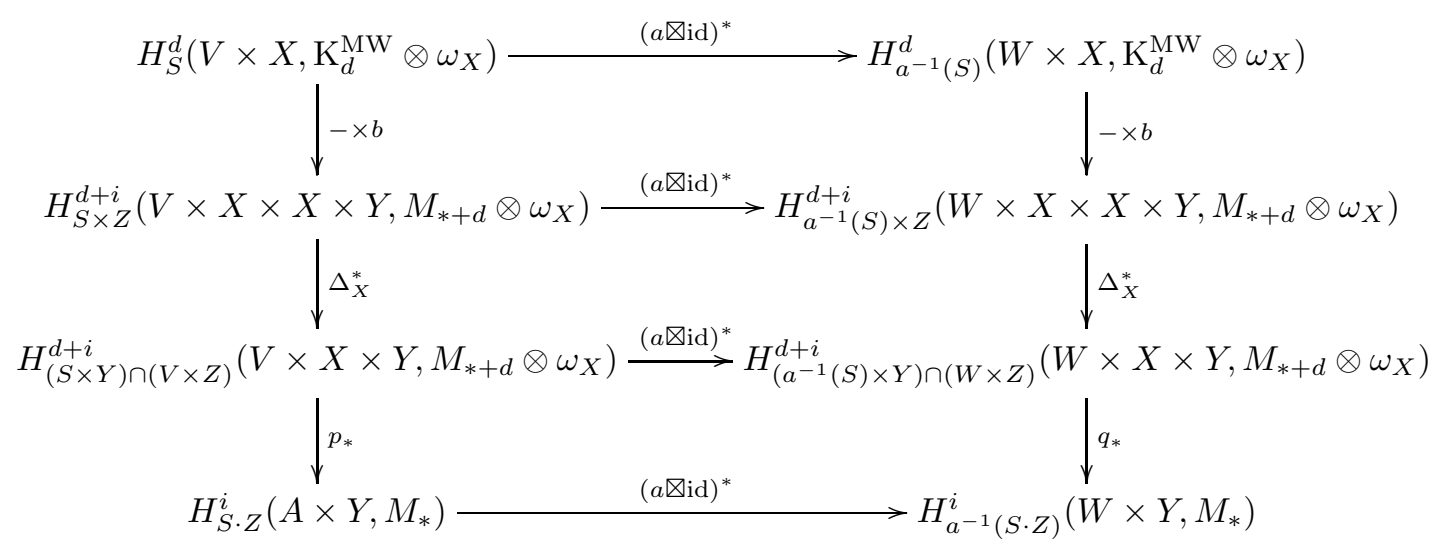

Here $p: V \times X \times Y \rightarrow V \times Y$ and $q: W \times X \times Y \rightarrow W \times Y$ are the projections. The top square commutes by Lemma 3.16, the middle square commutes since it consists of pullbacks, the bottom square commutes by Lemma 6.11 .

7.7. Lemma. In the notation of Definition 7.5 put $d^{\prime}=\operatorname{dim} Y$, let $L_{Y}$ be the total space of the canonical line bundle $\omega_{Y}$ and let $S^{\prime} \subset X \times Y$ be an admissible closed subset. Then the following diagram commutes.

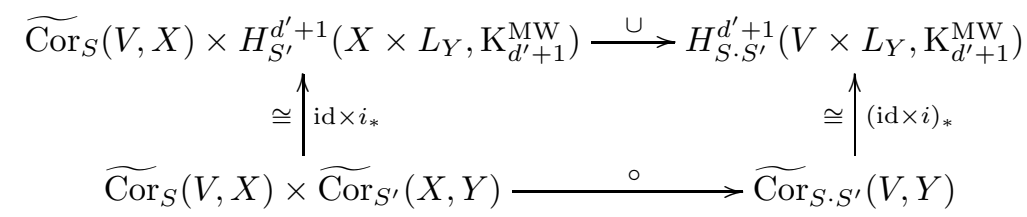

Here $i_{*}$ is the isomorphism from Remark 7.2 and $\circ$ is the composition of $M W$-correspondences introduced in $[4, \S 4.2]$

Proof. Recall that [4, Lemma 4.6] yields that for an open embedding $V_{0} \subset V$ the restriction

$$
\widetilde{\operatorname{Cor}}_{S \cdot S^{\prime}}(V, Y) \rightarrow \widetilde{\operatorname{Cor}}_{\left(S \cdot S^{\prime}\right) \cap\left(V_{0} \times Y\right)}\left(V_{0}, Y\right)
$$

is injective, thus it is sufficient to prove the claim for an open subset $V_{0}$. Note that

$$
\widetilde{\operatorname{Cor}}_{S \cap\left(V_{0} \times X\right)}\left(V_{0}, X\right)=\widetilde{\operatorname{Cor}}_{S \cap\left(V_{0} \times X_{0}\right)}\left(V_{0}, X_{0}\right)
$$

for every open subset $X_{0} \subset X$ such that $\pi\left(S \cap V_{0} \times X\right) \subset X_{0}$ for the projection $\pi: V \times X \rightarrow X$. Similarly

$$
\begin{aligned}
\widetilde{\operatorname{Cor}}_{S^{\prime} \cap\left(X_{0} \times Y\right)}\left(X_{0}, Y\right) & =\widetilde{\operatorname{Cor}}_{S^{\prime} \cap\left(X_{0} \times Y_{0}\right)}\left(X_{0}, Y_{0}\right), \\
\widetilde{\operatorname{Cor}}_{\left(S \cdot S^{\prime}\right) \cap\left(V_{0} \times Y\right)}\left(V_{0}, Y\right) & =\widetilde{\operatorname{Cor}}_{\left(S \cdot S^{\prime}\right) \cap\left(V_{0} \times Y_{0}\right)}\left(V_{0}, Y_{0}\right) .
\end{aligned}
$$

Thus taking $V_{0}$ to be sufficiently small, shrinking $X$ to $X_{0}$ and $Y$ to $Y_{0}$ accordingly and applying Lemma 5.9 we may assume that $X$ and $Y$ admit étale maps to affine spaces. 
Let $\theta: \omega_{Y} \stackrel{\simeq}{\rightarrow} \mathcal{O}_{Y}$ be the trivialization of $\omega_{Y}$ given by an étale map $Y \rightarrow \mathbb{A}_{k}^{d^{\prime}}$. Consider the following diagram.

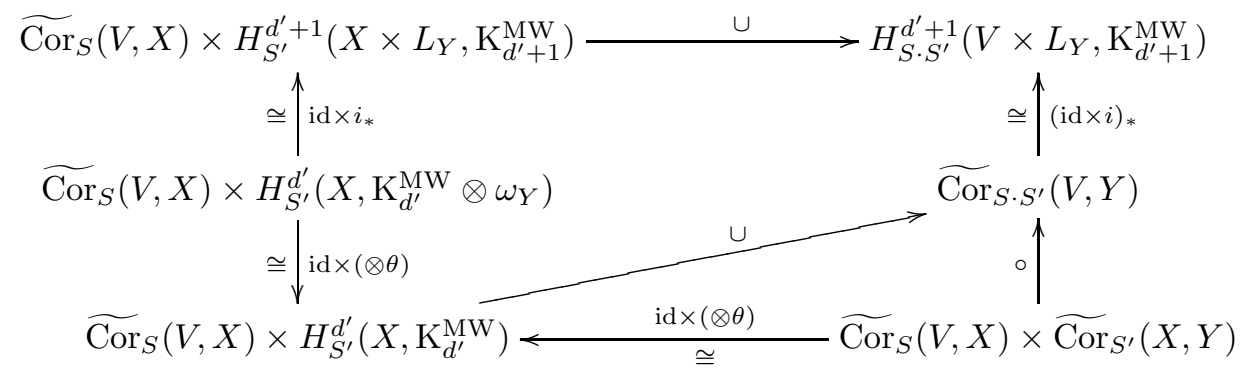

The bottom triangle commutes: compare the formula from Definition 7.5 with [4, §4.2] in the case of $\omega_{Y} \cong \mathcal{O}_{Y}$ (see also Remark 6.5). The commutativity of the upper half is straightforward, since $i_{*}$ commutes with all the involved pullbacks (Lemma 6.11), pushforwards (functoriality of pushforward maps) and external products (Lemma 6.12).

7.8. Proposition. The map $\mathcal{D}: \mathbb{Z F}_{*}(X, Y) \rightarrow \widetilde{\operatorname{Cor}}(X, Y)$ from Definition 7.3 gives rise to a functor

$$
\mathcal{D}: \mathbb{Z F}_{*}(k) \rightarrow \widetilde{\operatorname{Cor}_{k}} .
$$

Proof. Let $X, Y, V, W \in \mathrm{Sm}_{k}$ and $S_{1} \subset W \times X, S_{2} \subset X \times Y$ be admissible closed subsets. For $a \in \operatorname{Fr}_{n}(V, W)$ consider the following diagram.

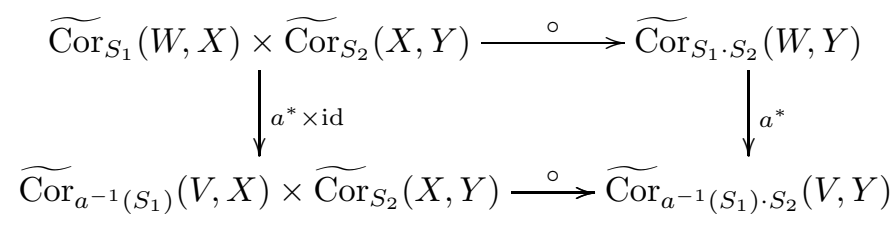

Applying the reasoning similar to the one used in the beginning of the proof of Lemma 7.7 we may assume that $X \rightarrow \operatorname{Spec} k$ admits a framed enhancement. Then the diagram commutes by Lemmas 7.6 and 7.7 .

Then for $b \in \mathbb{Z F}_{*}(Y, Z)$ and $a \in \mathbb{Z F}_{*}(X, Y)$ we have

$$
\mathcal{D}(b \circ a)=(b \circ a)^{*}\left(1_{Z}\right)=a^{*}\left(b^{*}\left(1_{Z}\right)\right)=a^{*}\left(\left(b^{*}\left(1_{Z}\right)\right) \circ 1_{Y}\right)=b^{*}\left(1_{Z}\right) \circ a^{*}\left(1_{Y}\right)=\mathcal{D}(b) \circ \mathcal{D}(a),
$$

Since $\mathcal{D}$ clearly respects the identity morphisms the claim follows.

\section{MilnoR-Witt tRAnSFERS ON HOMOTOPY MOdUlES}

8.1. Definition. Let $X, Y \in \mathrm{Sm}_{k}$ and $M_{*}$ be a homotopy module. Then for every admissible closed subset $S \subset Y \times X$ Definition 7.5 gives a pairing

$$
\widetilde{\operatorname{Cor}}_{S}(Y, X) \times M_{*}(X) \stackrel{\cup}{\rightarrow} M_{*}(Y)
$$

inducing a pairing

$$
\widetilde{\operatorname{Cor}}(Y, X) \times M_{*}(X) \stackrel{\cup}{\rightarrow} M_{*}(Y) .
$$


8.2. Lemma. In the notation of Definition 8.1 let $j: Y_{0} \rightarrow Y$ be an open embedding. Then the following diagram commutes.

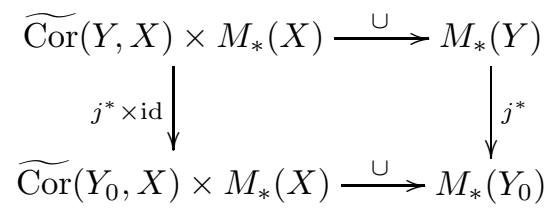

Proof. This follows from the fact that pullback along an open embedding commutes with external products (Lemma 3.16), pullbacks (functoriality of pullbacks) and pushforward maps (Lemma 6.4).

8.3. Lemma. In the notation of Definition 8.1 for $V \in \operatorname{Sm}_{k}$ and $a \in \operatorname{Fr}_{n}(V, Y)$ the following diagram commutes.

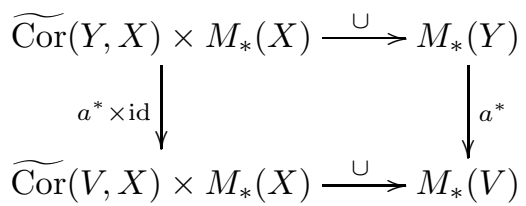

Proof. Recall that for every open subset $V_{0} \subset V$ the restriction $M_{*}(V) \rightarrow M_{*}\left(V_{0}\right)$ is injective. Hence by Lemma 8.2 it is sufficient to prove the statement for any open subset $V_{0}$ of $V$. Applying the reasoning similar to the one used in the beginning of the proof of Lemma 7.7 and shrinking $V, X$ and $Y$ we may assume that $X \rightarrow$ Spec $k$ admits a framed enhancement. Then the diagram commutes by Lemma 7.6.

8.4. Lemma. For $X \in \mathrm{Sm}_{k}$, a homotopy module $M_{*}$ and the identity morphism $1_{X} \in \widetilde{\operatorname{Cor}}(X, X)$ the following holds:

$$
\left(1_{X} \cup-\right)=\mathrm{id}: M_{*}(X) \rightarrow M_{*}(X) .
$$

Proof. Recall that for every open subset $X_{0} \subset X$ the restriction $M_{*}(X) \rightarrow M_{*}\left(X_{0}\right)$ is injective, then, applying Lemmas 8.2 and 5.9 we may assume that $X$ admits an étale map $\pi: X \rightarrow \mathbb{A}_{k}^{d}$. Consider the map

$$
\phi: X \times X \rightarrow \mathbb{A}_{k}^{d}, \quad \phi\left(x_{1}, x_{2}\right)=\pi\left(x_{1}\right)-\pi\left(x_{2}\right) .
$$

Since $\pi$ is étale, the zero set $Z(\phi)$ is $Z(\phi)=\Delta(X) \sqcup \mathcal{Z}^{\prime}$ for some closed $\mathcal{Z}^{\prime}$. Then

$$
a=\left(\Delta(X), U, \phi, p_{2}\right) \in \operatorname{Fr}_{0}(X \times X \stackrel{\Delta}{\longleftarrow} X)
$$

with $U=X \times X-\mathcal{Z}^{\prime}$ and $p_{1}, p_{2}: X \times X \rightarrow X$ being the projections is a framed enhancement of the diagonal morphism $X \stackrel{\Delta}{\rightarrow} X \times X$. Fix the trivialization of $\omega_{X}$ induced by $a$.

Recall that $1_{X}=\Delta_{*}(1)$, thus we need to check that for every $m \in M_{*}(X)$ one has

$$
p_{1 *}\left(\operatorname{id}_{X} \times \Delta\right)^{*}\left(\Delta_{*}(1) \times m\right)=m
$$

in the following diagram.

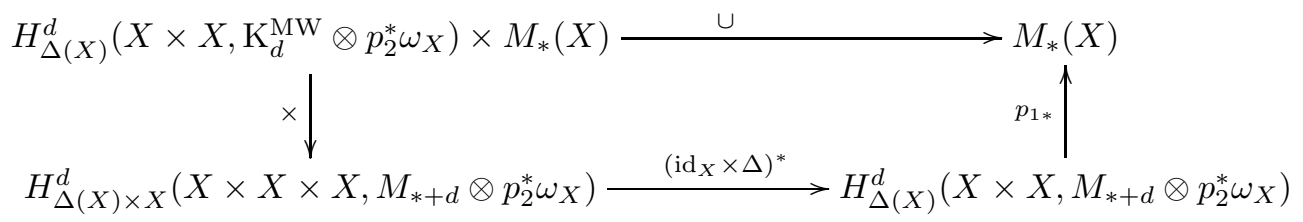


Lemma 6.10 yields that $\Delta_{*}(1)=a_{\Delta}^{*}(1)$ whence

$$
\begin{gathered}
\left(\operatorname{id}_{X} \times \Delta\right)^{*}\left(\Delta_{*}(1) \times m\right)=\left(\operatorname{id}_{X} \times \Delta\right)^{*}\left(a_{\Delta}^{*}(1) \times m\right)=a_{\Delta}^{*}(m)=\Delta_{*}(m), \\
p_{1 *}\left(\operatorname{id}_{X} \times \Delta\right)^{*}\left(\Delta_{*}(1) \times m\right)=p_{1 *} \Delta_{*}(m)=m .
\end{gathered}
$$

8.5. Lemma. In the notation of Definition 8.1 for $V \in \operatorname{Sm}_{k}$ and $\alpha \in \widetilde{\operatorname{Cor}}(V, Y)$ the following diagram commutes.

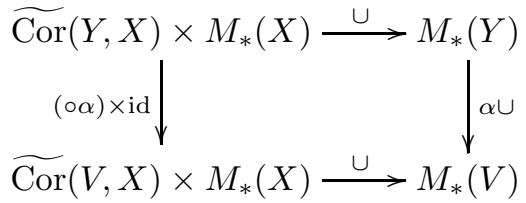

Proof. Applying the reasoning similar to the one in the beginning of the proof of Lemma 8.4 we may assume that $X$ and $Y$ admit étale morphisms to the affine spaces of respective dimension. Lemma 7.7 yields that $\circ$ can be interpreted in the terms of $\cup$ which is defined using external product, pullback and pushforward. A straightforward although rather lengthy computation shows that the claim follows from Lemmas 6.11 and 6.12 .

8.6. Definition. Let $\Pi_{*}^{\mathrm{MW}}(k)$ denote the category of homotopy modules with MW-transfers, i.e. the category of pairs $\left(M_{*}, \phi_{*}\right)$ where $M_{*}$ is a $\mathbb{Z}$-graded homotopy invariant sheaf with MWtransfers and $\phi_{i}: M_{i} \rightarrow\left(M_{i+1}\right)_{-1}$ are isomorphisms of sheaves with MW-transfers. We usually shorten the notation and refer to $\left(M_{*}, \phi_{*}\right)$ as $M_{*}$. Definition 7.3 (see also Proposition 7.8) gives rise to a functor

which descends to a functor

$$
\Phi^{\mathrm{MW}}: \mathrm{ShvAb}_{k}^{\mathrm{MW}} \rightarrow \mathrm{ShvAb}_{k}^{\mathrm{fr}}
$$

$$
\Phi^{\mathrm{MW}}: \Pi_{*}^{\mathrm{MW}}(k) \rightarrow \Pi_{*}^{\mathrm{fr}}(k) .
$$

Here $\operatorname{ShvAb}_{k}^{\mathrm{MW}}$ and $\mathrm{ShvAb}_{k}^{\mathrm{fr}}$ stand for the categories of sheaves with MW-transfers and $\mathbb{Z F}_{*}$ sheaves respectively. Definition 8.1 (see also Lemmas 8.4 and 8.5) gives rise to a functor

$$
\Psi^{\mathrm{MW}}: \Pi_{*}(k) \rightarrow \mathrm{ShvAb}_{k}^{\mathrm{MW}} .
$$

The functor $\Psi^{\mathrm{MW}}$ takes an isomorphism of graded sheaves $\phi_{*}: M_{*} \stackrel{\simeq}{\rightarrow}\left(M_{*+1}\right)_{-1}$ to an isomorphism of graded sheaves with MW-transfers, thus it descends to a functor

$$
\Psi^{\mathrm{MW}}: \Pi_{*}(k) \rightarrow \Pi_{*}^{\mathrm{MW}}(k) .
$$

8.7. Definition. For $X \in \mathrm{Sm}_{k}$ denote $\mathrm{H}_{0}^{\mathrm{MW}}(X)$ the sheaf associated with the presheaf

$$
U \mapsto \operatorname{coker}\left[\widetilde{\operatorname{Cor}}\left(U \times \mathbb{A}^{1}, X\right) \stackrel{i_{0}^{*}-i_{1}^{*}}{\longrightarrow} \widetilde{\operatorname{Cor}}(U, X)\right] .
$$

Here $i_{0}, i_{1}: U \rightarrow U \times \mathbb{A}^{1}$ are given by $i_{0}(u)=(u, 0)$ and $i_{1}(u)=(u, 1)$ respectively. Note that $\mathrm{H}_{0}^{\mathrm{MW}}(X)$ carries a canonical structure of a presheaf with MW-transfers.

8.8. Theorem. For $X \in \mathrm{Sm}_{k}$ the functor $\mathcal{D}: \mathbb{Z F}_{*}(k) \rightarrow \widetilde{\operatorname{Cor}}_{k}$ from Proposition 7.8 induces an isomorphism of sheaves

$$
f: \mathrm{H}_{0}^{\mathbb{Z F}}(X) \stackrel{\simeq}{\rightarrow} \mathrm{H}_{0}^{\mathrm{MW}}(X) .
$$

Proof. Let $\overline{\mathrm{id}}_{X} \in \mathrm{H}_{0}^{\mathbb{Z F}}(X)(X)$ be the image of the identity id $X \in \mathbb{Z} \mathrm{F}(X)(X)$. Then Definition 8.1 yields a morphism

$$
\widetilde{\operatorname{Cor}}(-, X) \underset{28}{\stackrel{\cup \overline{\mathrm{id}}_{X}}{\longrightarrow}}\left(\mathrm{H}_{0}^{\mathbb{Z} \mathrm{F}}(X)_{*}\right)_{0} .
$$


Here $\mathrm{H}_{0}^{\mathbb{Z F}}(X)_{*}$ is the framed homotopy module given by Definition 3.8. Since the right-hand side is homotopy invariant, it descends to a morphism

$$
g: \mathrm{H}_{0}^{\mathrm{MW}}(X) \rightarrow \mathrm{H}_{0}^{\mathbb{Z F}}(X)_{0}=\mathrm{H}_{0}^{\mathbb{Z F}}(X) .
$$

We need to check that $f$ and $g$ are inverse to each other. For $U \in \operatorname{Sm}_{k}, \bar{a} \in \mathrm{H}_{0}^{\mathbb{Z F}}(X)(U)$ being the image of $a \in \mathbb{Z} \mathrm{F}(X)(U)$ Lemmas 8.3 and 8.4 yield

$$
\left(g_{U} \circ f_{U}\right)(\bar{a})=a^{*}\left(1_{X}\right) \cup \overline{\mathrm{id}}_{X}=a^{*}\left(1_{X} \cup \overline{\mathrm{id}}_{X}\right)=a^{*}\left(\overline{\mathrm{id}}_{X}\right)=\bar{a}
$$

whence $g \circ f=$ id. For $\alpha \in \mathrm{H}_{0}^{\mathrm{MW}}(X)(U)$ we have

$$
\left(f_{U} \circ g_{U}\right)(\alpha)=\left(\alpha \cup \overline{\mathrm{id}}_{X}\right)^{*}\left(1_{X}\right)=\left(f_{U}\left(\alpha \cup \overline{\mathrm{id}}_{X}\right)\right) \circ 1_{X}=\alpha \circ f_{U}\left(\overline{\mathrm{id}}_{X}\right)=\alpha .
$$

Here the second equality follows from the fact that the framed structure induced by $\mathcal{D}$ on $\mathrm{H}_{0}^{\mathrm{MW}}(X)$ agrees with the structure of a presheaf with MW-transfers. The third equality follows from the fact that $f$ can be promoted to a morphism of presheaves with MW-transfers with $\mathrm{H}_{0}^{\mathbb{Z F}}(X)$ being endowed with the structure of a presheaf with $\mathrm{MW}$-transfers applying $\Psi^{\mathrm{MW}}$ to $\mathrm{H}_{0}^{\mathbb{Z F}}(X)_{*}$. Thus $f \circ g=$ id and the claim follows.

8.9. Corollary. For $X \in \mathrm{Sm}_{k}$ there is a canonical isomorphism of sheaves

$$
\pi_{0}\left(\Sigma_{\mathbb{P}^{1}}^{\infty} X_{+}\right)_{0} \cong \mathrm{H}_{0}^{\mathrm{MW}}(X) .
$$

Proof. Follows from Theorem 8.8 combined with the isomorphism $\pi_{0}\left(\Sigma_{\mathbb{P} 1}^{\infty} X_{+}\right)_{0} \cong \mathrm{H}_{0}^{\mathbb{Z F}}(X)$ given by [9, Theorem 11.1 and Corollary 11.3].

8.10. Remark. The above corollary provides a reasonable description for the zeroth stable motivic homotopy sheaf of $X$. A similar computation with $X$ being a smooth projective variety was obtained by different means in [3, Theorem 4.3.1] and [1, Theorem 1.2], see also [1, Theorem 1.3] for the case of a smooth curve.

8.11. Corollary. Let $M$ be a homotopy invariant stable $\mathbb{Z F}_{*}$-sheaf. Then $M$ admits a unique structure of a presheaf with $M W$-transfers compatible with the framed structure.

Proof. To give $M$ a structure of a presheaf with MW-transfers compatible with the framed structure is the same thing as to construct for every $X \in \mathrm{Sm}_{k}$ a map $\phi_{X}$ such that the following diagram commutes

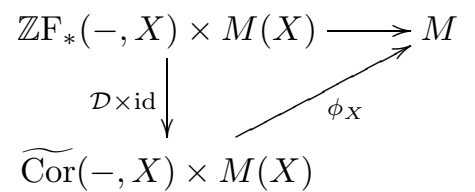

and check associativity relation. Here the horizontal arrow is given by the framed structure and the vertical one is induced by the functor $\mathcal{D}$. Since $M$ is homotopy invariant and stable then it is the same as to construct $\bar{\phi}_{X}$ for the following diagram.

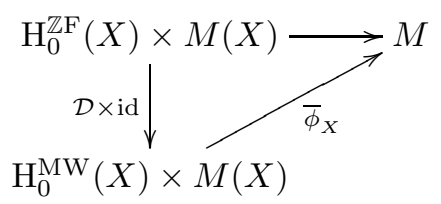

Here the vertical morphism is an isomorphism by Theorem 8.8 so the claim follows. 
8.12. Theorem. The forgetful functors $\Phi^{\mathrm{fr}}$ and $\Phi^{\mathrm{MW}}$ from Definitions 3.5 and 8.6 respectively establish equivalences of categories

$$
\Pi_{*}^{\mathrm{MW}}(k) \stackrel{\Phi^{\mathrm{MW}}}{\simeq} \Pi_{*}^{\mathrm{fr}}(k) \underset{\simeq}{\stackrel{\Phi^{\mathrm{fr}}}{\longrightarrow}} \Pi_{*}(k) .
$$

Proof. The functor $\Phi^{\text {fr }}$ is an equivalence by Proposition 3.11. Lemma 8.3 yields that

$$
\Phi^{\mathrm{MW}} \circ \Psi^{\mathrm{MW}} \circ \Phi^{\mathrm{fr}}=\mathrm{id} .
$$

Then it is sufficient to check that if $\Phi^{\mathrm{MW}} \circ \Phi^{\mathrm{fr}}\left(M_{*}\right) \cong \Phi^{\mathrm{MW}} \circ \Phi^{\mathrm{fr}}\left(M_{*}^{\prime}\right)$ then $M_{*} \cong M_{*}^{\prime}$, i.e. that a framed homotopy module carries a unique structure of a homotopy module with MWtransfers compatible with the framed structure. The reasoning is the same as in the proof of Corollary 8.11. The structure of a presheaf with MW-transfers is given by morphisms of presheaves $\widetilde{\operatorname{Cor}}(-, X) \times M_{*}(X) \rightarrow M_{*}$ which descend to

$$
\mathrm{H}_{0}^{\mathrm{MW}}(X) \times M_{*}(X) \rightarrow M_{*} .
$$

The latter one is unique (compatible with the framed structure) since Theorem 8.8 yields an isomorphism of sheaves $\mathrm{H}_{0}^{\mathrm{MW}}(X) \cong \mathrm{H}_{0}^{\mathbb{Z F}}(X)$ and the morphism

$$
\mathrm{H}_{0}^{\mathrm{MW}}(X) \times M_{*}(X) \cong \mathrm{H}_{0}^{\mathbb{Z F}}(X) \times M_{*}(X) \rightarrow M_{*}
$$

is induced by the framed structure $\mathbb{Z} \mathrm{F}(X) \times M_{*}(X) \rightarrow M_{*}$.

8.13. Remark. Recall that the category of homotopy modules is equivalent to the heart of the homotopy $t$-structure on the stable $\mathbb{A}^{1}$-derived category $D_{\mathbb{A}^{1}}(k)$ (see, for example, [3, Theorem 3.3.3]). In a similar way one can show that the category of homotopy modules with MWtransfers is equivalent to the heart of the homotopy $t$-structure on the category of MW-motives $\widetilde{\mathrm{DM}}(k)$ introduced in [7].

8.14. Corollary. The forgetful functor $\widetilde{\mathrm{DM}}(k) \rightarrow D_{\mathbb{A}^{1}}(k)$ induces an equivalence on the hearts of the homotopy t-structures.

Proof. Follows from Theorem 8.12 .

\section{REFERENCES}

[1] A. Ananyevskiy. On the zeroth stable $\mathbb{A}^{1}$-homotopy group of a smooth curve. Published online in $J$. Pure Appl. Algebra, doi:10.1016/j.jpaa.2017.12.001.

[2] A. Ananyevskiy, G. Garkusha, I. Panin. Cancellation theorem for framed motives of algebraic varieties. Preprint, arXiv:1601.06642.

[3] A. Asok, C. Haesemeyer. The 0-th stable $\mathbb{A}^{1}$-homotopy sheaf and quadratic zero cycles. Preprint, arXiv:1108.3854.

[4] B. Calmès, J. Fasel. The category of finite MW-corrsespondences. Preprint, arXiv:1412.2989.

[5] B. Calmès, J. Fasel. A comparison theorem for MW-motivic cohomology. Preprint, arXiv:1708.06100.

[6] J.-L. Colliot-Thélène, R. Hoobler, B. Kahn. The Bloch-Ogus-Gabber theorem. Algebraic K-theory (Toronto, ON, 1996), 31-94, Fields Inst. Commun., 16, Amer. Math. Soc., Providence, RI, 1997.

[7] F. Déglise, J. Fasel. MW-motivic complexes. Preprint, arXiv:1708.06095

[8] J. Fasel, P. Østvaer. A cancellation theorem for Milnor-Witt correspondences. Preprint, arXiv:1708.06098.

[9] G. Garkusha, I. Panin. Framed motives of algebraic varieties (after V. Voevodsky) Preprint, arXiv:1409.4372

[10] G. Garkusha, I. Panin. Homotopy invariant presheaves with framed transfers Preprint, arXiv:1504.00884

[11] H. Lindel. On the Bass-Quillen conjecture concerning projective modules over polynomial rings. Invent. Math. 65 (1981/82), no. 2, 319-323.

[12] C. Mazza, V. Voevodsky, C. Weibel. Lecture notes on motivic cohomology. Clay Mathematics Monographs, vol. 2, American Mathematical Society, Providence, RI, 2006

[13] F. Morel. $\mathbb{A}^{1}$-algebraic topology over a field. Lecture Notes in Mathematics, 2052. Springer, Heidelberg, 2012.

[14] F. Morel. An introduction to $\mathbb{A}^{1}$-homotopy theory. Contemporary developments in algebraic $K$-theory, 357 441. 
[15] A. Neshitov. Framed correspondences and the Milnor-Witt K-theory, J. Inst. Math. Jussieu, available on CJO2016. doi:10.1017/S1474748016000190.

[16] V. Voevodsky. Notes on framed correspondences. Unpublished, 2001, available at math.ias.edu/vladimir/files/framed.pdf

[17] C. Weibel. The $K$-book. An introduction to algebraic $K$-theory. Graduate Studies in Mathematics, 145. American Mathematical Society, Providence, RI, 2013.

St. Petersburg Department, Steklov Math. Institute, Fontanka 27, St. Petersburg 191023 Russia, and Chebyshev Laboratory, St. Petersburg State University, 14th Line V.O., 29B, St. Petersburg 199178 RUSSIA

E-mail address: alseang@gmail.com

Department of Mathematics, University of Southern California, 3620 S. Vermont ave, Los Angeles, CA, 90089

E-mail address: neshitov@usc.edu 